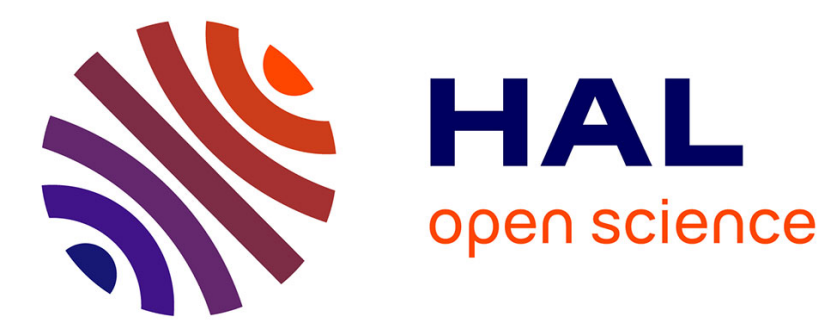

\title{
Sur les équations vérifiées par les invariants des fibrés exceptionnels
}

\author{
Jean-Marc Drézet
}

\section{To cite this version:}

Jean-Marc Drézet. Sur les équations vérifiées par les invariants des fibrés exceptionnels. Forum Mathematicum, 1996, 8, pp.237-265. hal-00742352

\section{HAL Id: hal-00742352 \\ https://hal.science/hal-00742352}

Submitted on 16 Oct 2012

HAL is a multi-disciplinary open access archive for the deposit and dissemination of scientific research documents, whether they are published or not. The documents may come from teaching and research institutions in France or abroad, or from public or private research centers.
L'archive ouverte pluridisciplinaire HAL, est destinée au dépôt et à la diffusion de documents scientifiques de niveau recherche, publiés ou non, émanant des établissements d'enseignement et de recherche français ou étrangers, des laboratoires publics ou privés. 


\title{
SUR LES ÉQUATIONS VÉRIFIÉES PAR LES INVARIANTS DES FIBRÉS EXCEPTIONNELS
}

\author{
JEAN-MARC DRÉZET
}

SOMMAIRE

0. Introduction 1

\begin{tabular}{lll}
\hline 1. & Préliminaires & 7
\end{tabular}

2 2. Les variétés $Z(\phi) \quad 8$

3. Courbes planes associées aux bases d'hélices de fibrés exceptionnels 16

4. Equations vérifiées par les invariants des fibrés exceptionnels 20

Références 25

\section{INTRODUCTION}

\subsection{Hélices de fibrés exceptionnels}

Soit $E$ un fibré vectoriel algébrique sur une variété algébrique projective complexe $X$. On dit que $E$ est exceptionnel si on a $\operatorname{End}(E) \simeq \mathbb{C}$ et $\operatorname{Ext}^{i}(E, E)=0$ pour $i \geq 1$. Sur certaines variétés $X$, les espaces projectifs par exemple, il existe des suites parti-culières de fibrés exceptionnels $\left(E_{i}\right)_{i \in \mathbb{Z}}$, appelées hélices.

Les fibrés exceptionnels ont été introduits et étudiés sur $\mathbb{P}_{2}$ dans [3], [1], puis leur généralisation sur $\mathbb{P}_{n}$ a été faite dans [5]. Le cas de $\mathbb{P}_{1} \times \mathbb{P}_{1}$ a été traité dans [8], celui de certaines surfaces dans [4], et celui des surfaces de Hirzebruch et de certains volumes de Fano dont $\mathbb{P}_{3}$ dans [6], [7.

Une hélice de fibrés exceptionnels $\left(E_{i}\right)_{i \in \mathbb{Z}}$ sur $\mathbb{P}_{n}$ est périodique, c'est-à-dire que pour tout entier $i$ on a $E_{i+n+1} \simeq E_{i}(n+1)$. Il suffit donc de connaître $n+1$ fibrés exceptionnels consécutifs de l'hélice pour la connaître tout entière. On dit qu'une suite $\left(E_{i}, E_{i+1}, \ldots, E_{i+n+1}\right)$ est une base de l'hélice $\left(E_{i}\right)_{i \in \mathbb{Z}}$. On notera $B\left(\mathbb{P}_{n}\right)$ l'ensemble des bases d'hélices de $\mathbb{P}_{n}$. On appelle paire exceptionnelle un couple du type $\left(E_{i}, E_{i+1}\right)$.

Les rangs et classes de Chern des fibrés exceptionnels d'une base d'hélice vérifient certaines équations. Par exemple, sur $\mathbb{P}_{2}$, si $\left(E_{0}, E_{1}, E_{2}\right)$ est une base d'hélice, on a

$$
r g\left(E_{0}\right)^{2}+r g\left(E_{1}\right)^{2}+r g\left(E_{2}\right)^{2}=3 r g\left(E_{0}\right) r g\left(E_{1}\right) r g\left(E_{2}\right) .
$$

C'est l'équation de Markov (cf. [5]). Sur $\mathbb{P}_{3}$, Nogin a trouvé aussi certaines relations (cf. [6] et [7]). 
On montre ici que d'une certaine manière, les seules relations algébriques que l'on peut obtenir entre les rangs et classes de Chern des fibrés exceptionnels d'une base d'hélice peuvent se déduire des relations "évidentes".

\subsection{Equations algébriques liant les rangs et les classes de Chern des fibrés excep- tionnels d'une base d'hélice sur $\mathbb{P}_{3}$}

Pour tout faisceau cohérent $\mathcal{E}$ sur une variété projective $X$ de dimension $n$, on pose

$$
\chi(\mathcal{E})=\sum_{0 \leq i \leq n}(-1)^{i} \operatorname{dim}\left(H^{i}(X, \mathcal{E})\right),
$$

qui est la caractéristique d'Euler de $\mathcal{E}$. Soit $\left(E_{i}\right)_{0 \leq i \leq 3}$ une base d'hélice de fibrés exceptionnels sur $\mathbb{P}_{3}$. On a alors

$$
\begin{aligned}
& \chi\left(E_{i}, E_{j}\right)=0 \quad \text { si } i>j, \\
& =1 \text { si } i=j \text {, }
\end{aligned}
$$

ce qui, à l'aide du théorème de Riemann-Roch, s'écrit sous la forme

$$
C\left(r g\left(E_{i}\right), r g\left(E_{j}\right),\left(c_{k}\left(E_{i}\right)\right)_{1 \leq k \leq 3},\left(c_{k}\left(E_{j}\right)\right)_{1 \leq k \leq 3}\right)=\delta_{i j},
$$

$C$ étant un polynôme à coefficients rationnels.

Soient $X$ une variété algébrique projective lisse, $E$ un fibré vectoriel de rang $r>0$ sur $X$. On définit au chapitre 1 de nouveaux invariants

$$
\Delta_{i}(E) \in A^{i}(E) \otimes \mathbb{Q}, 1 \leq i \leq \operatorname{dim}(X) .
$$

Ils ont l'avantage de transformer les produits tensoriels en sommes, et $\Delta_{1}(E)$ est la pente de $E$, tandis que $\Delta_{2}(E)$ est le discriminant de $E$. Les relations précédentes s'écrivent avec ces invariants

$$
Q\left(\left(\Delta_{k}\left(E_{i}\right)\right)_{1 \leq k \leq 3},\left(\Delta_{k}\left(E_{j}\right)\right)_{1 \leq k \leq 3}\right)=0
$$

si $0 \leq j<i \leq 3$, et

$$
\operatorname{rg}\left(E_{i}\right)^{2} \cdot\left(1-4 \Delta_{2}\left(E_{i}\right)\right)=1,0 \leq i \leq 3
$$

$Q$ étant le polynôme

$$
Q\left(\left(Y_{k}\right)_{1 \leq k \leq 3},\left(Z_{k}\right)_{1 \leq k \leq 3}\right)=Z_{3}-Y_{3}-\left(Z_{1}-Y_{1}+2\right)\left(Y_{2}+Z_{2}\right)+\left(\begin{array}{c}
Z_{1}-Y_{1}+3 \\
3
\end{array}\right) .
$$

On note $\mathcal{I}$ l'idéal de l'anneau de polynômes

$$
R=\mathbb{C}\left[X_{0}, X_{1}, X_{2}, X_{3},\left(\left(\Delta_{p q}\right)_{1 \leq p \leq 3}\right)_{0 \leq q \leq 3}\right]
$$

engendré par les

$$
\chi_{i j}=Q\left(\left(\Delta_{k i}\right)_{1 \leq k \leq 3},\left(\Delta_{k j}\right)_{1 \leq k \leq 3}\right)
$$

avec $0 \leq j<i \leq 3$ et les

$$
\chi_{i i}=X_{i} \cdot\left(1-4 \Delta_{2 i}\right)-1 \text {. }
$$

Tous les éléments de $\mathcal{I}$ s'annulent donc sur les carrés des rangs et les $\Delta_{i}$ des fibrés exceptionnels d'une base d'hélice .

On a réciproquement, dans le cas de $\mathbb{P}_{3}$ le 
Théorème $\mathbf{A}$ - Si $P$ est un élément de $R$ tel que pour toute base d'hélice $\left(E_{0}, E_{1}, E_{2}, E_{3}\right)$ on ait

$$
P\left(\left(\operatorname{rg}\left(E_{i}\right)^{2}\right)_{0 \leq i \leq 3},\left(\left(\Delta_{k}\left(E_{i}\right)\right)_{1 \leq k \leq 3}\right)_{0 \leq i \leq 3}\right)=0,
$$

alors il existe un entier $p>0$ tel que $P^{p}$ soit dans $\mathcal{I}$.

Pour obtenir ce résultat, on construit une variété algébrique $Z$ telle que les bases d'hélices de $\mathbb{P}_{3}$ soient des points fermés de $Z$, et que les fonctions définies sur $B\left(\mathbb{P}_{3}\right)$

$$
\begin{gathered}
\left(E_{0}, E_{1}, E_{2}, E_{3}\right) \longmapsto r g\left(E_{i}\right)^{2}, \quad 0 \leq i \leq 3, \\
\left(E_{0}, E_{1}, E_{2}, E_{3}\right) \longmapsto \Delta_{k}\left(E_{i}\right), \quad 1 \leq k \leq 3,0 \leq i \leq 3,
\end{gathered}
$$

se prolongent en des fonctions rationnelles sur $Z$. On montre ensuite que la plus petite sousvariété fermée de $Z$ contenant $B\left(\mathbb{P}_{3}\right)$ est $Z$ et le théorème $\mathrm{A}$ s'en déduit aisément.

On donne deux constructions de la variété $Z$, l'une basée sur le groupe de Grothendieck de $\mathbb{P}_{3}$, l'autre étant plus géométrique. Bien sûr il est théoriquement possible de prendre pour $Z$ la sous-variété de $\mathbb{C}^{16}$ définie par l'idéal $\mathcal{I}$, mais cette variété est a priori plus difficile à manier.

La démonstration du théorème $\mathrm{A}$ fournit des résultats intermédiaires intéressants, concernant les équations algébriques vérifiées par les rangs et $\Delta_{i}$ des fibrés exceptionnels d'une paire exceptionnelle, ou par le rang et les $\Delta_{i}$ des fibrés exceptionnels. Plus précisément, soient

$$
R^{\prime}=\mathbb{C}\left[R_{0}, R_{1},\left(\left(\Delta_{p q}\right)_{1 \leq p \leq 3}\right)_{0 \leq q \leq 1}\right] \quad \text { et } \quad R^{\prime \prime}=\mathbb{C}\left[R_{0},\left(\Delta_{p 0}\right)_{1 \leq p \leq 3}\right],
$$

$\mathcal{I}^{\prime}$ l'idéal de $R^{\prime}$ engendré par $\chi_{00}\left(R_{0}^{2}, \Delta_{20}\right), \chi_{10}, \chi_{11}\left(R_{1}^{2}, \Delta_{21}\right)$ et $\mathcal{I}^{\prime \prime}$ l'idéal de $R^{\prime \prime}$ engendré par $\chi_{00}\left(R_{0}^{2}, \Delta_{20}\right)$. Alors on a le

Théorème $\mathbf{B}$ - Tout élément de $R^{\prime}$ s'annulant sur les rangs et $\Delta_{i}$ des fibrés exceptionnels de toutes les paires exceptionnelles appartient à $\mathcal{I}^{\prime}$.

et le

Théorème $\mathbf{C}$ - Tout élément de $R^{\prime \prime}$ s'annulant sur le rang et les $\Delta_{i}$ de tous les fibrés exceptionnels appartient à $\mathcal{I}^{\prime \prime}$.

Ces résultats sont discutés au $§ 0.5$.

\subsection{Construction de la variété $Z$}

Soient $K\left(\mathbb{P}_{3}\right)$ le groupe de Grothendieck de $\mathbb{P}_{3}$ et $W_{K}=K\left(\mathbb{P}_{3}\right) \otimes \mathbb{C}$. Cet espace vectoriel est muni de la forme bilinéaire $\chi(x, y)$ définie par

$$
\chi\left([E],\left[E^{\prime}\right]\right)=\chi\left(E^{*} \otimes E^{\prime}\right),
$$

pour tous fibrés vectoriels $E, E^{\prime}$ sur $\mathbb{P}_{3},[E],\left[E^{\prime}\right]$ désignant leurs classes dans $K\left(\mathbb{P}_{3}\right)$. Si $\left(E_{0}, E_{1}, E_{2}, E_{3}\right)$ est une base d'hélice de fibrés exceptionnels, et si $e_{i}=\left[E_{i}\right]$ pour $0 \leq i \leq 3$, $\left(e_{0}, e_{1}, e_{2}, e_{3}\right)$ est une base semi-orthogonale de $W_{K}$, c'est-à-dire que la matrice de $\chi$ dans cette base est triangulaire supérieure unipotente. Il en découle que si

$$
u=\mathbb{C} . e_{0} \oplus \mathbb{C} . e_{1} \quad \text { et } \quad v=\mathbb{C} . e_{1} \oplus \mathbb{C} . e_{2},
$$


on a

$$
u \cap v \neq\{0\} \quad \text { et } \quad u^{\perp} \cap v \neq\{0\}
$$

$u^{\perp}$ désignant le plan de $W_{K}$ constitué des $x$ tels que $\chi(x, z)=0$ pour tout $z$ dans $u$. Soient

$$
\phi_{K}: W_{K} \longrightarrow W_{K}^{*}
$$

l'isomorphisme linéaire tel que $\chi(x, y)=x \cdot \phi_{k}(y)$ pour tous $x, y$ dans $W_{K}$, et $Z\left(\phi_{k}\right)$ la variété des couples $(u, v)$ de plans de $W_{K}$ tels que $u \cap v \neq\{0\}$ et $u^{\perp} \cap v \neq\{0\}$. Alors on peut voir $\left(e_{0}, e_{1}, e_{2}, e_{3}\right)$ comme un point de $Z\left(\phi_{k}\right)$, c'est-à-dire qu'on a défini une application

$$
\Lambda_{K}: B\left(\mathbb{P}_{3}\right) \longrightarrow Z\left(\phi_{k}\right)
$$

On étudie plus généralement au chapitre 2 les variétés $Z(\phi), \phi$ étant un isomorphisme linéaire $W \longrightarrow W^{*}$. On peut définir des automorphismes de $Z(\phi)$, appelés mutations, qui généralisent les mutations des hélices de fibrés exceptionnels, et qui ont les mêmes propriétés formelles.

On peut donner une description géométrique de certaines variétés $Z(\phi)$ au moyen de coniques de $\mathbb{P}_{2}$. Plus précisément, on suppose que $W$ est un sous-espace vectoriel de $H^{0}\left(\mathbb{P}_{2}, \mathcal{O}(2)\right)$ et on considère un morphisme rationnel

$$
\sigma: \mathbb{P}_{2} \longrightarrow \mathbb{P}(W)
$$

tel que $\sigma^{*}(\mathcal{O}(1)) \simeq \mathcal{O}(2)$, que $\sigma^{*}: W^{*} \longrightarrow H^{0}\left(\mathbb{P}_{2}, \mathcal{O}(2)\right)$ induise un isomorphisme $W^{*} \simeq W$ et que pour tout point $P$ de $\mathbb{P}_{2}$ où $\sigma$ est défini, on ait $P \in \sigma(P), \sigma(P)$ étant vu comme une conique de $\mathbb{P}_{2}$. On verra alors qu'il n'y a que trois choix possibles pour $W$, les plus intéressants étant le cas où $W$ correspond aux coniques passant par deux points fixes de $\mathbb{P}_{2}$, et celui où $W$ correspond aux coniques passant par un point fixe de $\mathbb{P}_{2}$ et tangentes à une droite fixe en ce point. Dans ces cas, $\sigma$ est un isomorphisme birationnel de $\mathbb{P}_{2}$ sur la quadrique $Q(\phi)$ de $\mathbb{P}(W)$ constituée des $x$ tels que $x . \phi(x)=0$. Un plan général de $\mathbb{P}(W)$ s'écrit

$$
u=\sigma\left(P_{0}\right) \wedge \sigma\left(P_{1}\right)
$$

$P_{0}, P_{1}$ étant des points de $\mathbb{P}_{2}$, et on a

$$
u^{\perp}=\sigma\left(P_{2}\right) \wedge \sigma\left(P_{3}\right),
$$

$P_{2}, P_{3}$ étant les points d'intersection de $\sigma\left(P_{0}\right)$ et $\sigma\left(P_{1}\right)$ autres que les points de base de $W$. On voit donc qu'un point général de $Z(\phi)$ s'interprète comme une configuration de quatre coniques vérifiant certaines propriétés.

On retrouve une description de ce type en considérant certaines courbes planes associées aux hélices de fibrés exceptionnels (cf. §0.4). L’utilisation possible de telles courbes est évoquée au $\S 0.5$.

\subsection{Courbes planes associées aux hélices de fibrés exceptionnels}

A tout fibré vectoriel non nul $E$ sur l'espace projectif $\mathbb{P}_{3}$ on associe le point $\left(\Delta_{1}(E), \Delta_{2}(E), \Delta_{3}(E)\right)$ de $\mathbb{R}^{3}$. On note $H$ le plan de $\mathbb{R}^{3}$ d'équation $\Delta_{2}=\frac{1}{4}$, qui semble jouer sur $\mathbb{P}_{3}$ un rôle analogue à celui de la droite d'équation $\Delta_{2}=\frac{1}{2} \quad$ sur $\mathbb{P}_{2}$ (cf. [3]). 
A tout fibré exceptionnel $E$ sur $\mathbb{P}_{3}$ on associe une courbe $C(E)$ de $H$, qu'on obtient de la manière suivante : si $U$ est un fibré vectoriel sur $\mathbb{P}_{3}$ d'invariants $\Delta_{1}, \Delta_{2}, \Delta_{3}$, l'expression

$$
\frac{\chi\left(U^{*} \otimes E\right)}{r g\left(U^{*} \otimes E\right)}
$$

est un polynôme en $\Delta_{1}, \Delta_{2}, \Delta_{3}$, qui est l'équation d'une hypersurface cubique de $\mathbb{R}^{3}$, dont l'intersection avec $H$ est $C(E)$. Son équation est de degré 3 , le terme de degré 3 étant $-\frac{1}{6} \Delta_{1}^{3}$. Moyennant un changement de coordonnées sur $H$ et en plongeant celui-ci dans $\mathbb{P}_{2}$, on associe maintenant à $E$ une conique de $\mathbb{P}_{2}$. On voit aisément que toutes ces coniques passent par un point fixe $Q$ de $\mathbb{P}_{2}$ et sont tangentes à une droite fixe $l$ de $\mathbb{P}_{2}$ en $Q$. Soit $W$ le sous-espace vectoriel de $H^{0}\left(\mathbb{P}_{2}, \mathcal{O}(2)\right)$ correspondant aux coniques passant par $Q$ et tangentes à $l$ en $Q$. A toute paire exceptionnelle $\left(E_{0}, E_{1}\right)$ on associe le plan $\gamma\left(E_{0}, E_{1}\right)$ de $W$ engendré par les coniques associées à $E_{0}$ et $E_{1}$. On montre alors que l'application qui associe à une base d'hélice $\left(E_{0}, E_{1}, E_{2}, E_{3}\right)$ le couple de plans $\left(\gamma\left(E_{0}, E_{1}\right), \gamma\left(E_{1}, E_{2}\right)\right)$ est en fait à valeurs dans une variété $Z(\phi)$ convenable, le morphisme $\phi$ étant comme on s'en doute équivalent à $\phi_{K}$ modulo un isomorphisme $W \simeq W_{K}$. Les deux points de l'intersection $Q(\phi) \cap \gamma\left(E_{0}, E_{1}\right)$ s'interprètent comme des coniques limites : en effet il existe une suite $\left(F_{i}\right)_{i \in \mathbb{Z}}$ de fibrés exceptionnels telle que

$$
F_{0}=E_{0}, F_{1}=E_{1},
$$

et que pour tout entier $i$ on ait une suite éxacte

$$
0 \longrightarrow F_{i-1} \longrightarrow F_{i} \otimes \operatorname{Hom}\left(F_{i}, F_{i+1}\right) \longrightarrow F_{i+1} \longrightarrow 0
$$

dont le second morphisme est l'évaluation, et $Q(\phi) \cap \gamma\left(E_{0}, E_{1}\right)$ est constitué des limites de coniques associées à $F_{i}$ lorsque $i$ tend vers $+\infty$ ou $-\infty$.

\subsection{Remarques}

1) L'équation la plus importante vérifiée par les rangs et classes de Chern des fibrés exceptionnels d'un base d'hélice, donnée dans [7], est la suivante : pour toute base d'hélice $\left(E_{0}, E_{1}, E_{2}, E_{3}\right)$, on a

$$
c_{1}\left(E_{1}\right) \cdot r g\left(E_{0}\right)-c_{1}\left(E_{0}\right) \cdot r g\left(E_{1}\right)=c_{1}\left(E_{3}\right) \cdot r g\left(E_{2}\right)-c_{1}\left(E_{2}\right) \cdot r g\left(E_{3}\right),
$$

ce qui s'écrit aussi

$$
r g\left(E_{1}\right) \cdot r g\left(E_{0}\right) \cdot\left(\Delta_{1}\left(E_{1}\right)-\Delta_{1}\left(E_{0}\right)\right)=r g\left(E_{3}\right) \cdot r g\left(E_{2}\right) \cdot\left(\Delta_{1}\left(E_{3}\right)-\Delta_{1}\left(E_{2}\right)\right) .
$$

Cependant, on peut montrer que l'équation précédente ne peut pas se déduire des équations $\chi\left(E_{i}, E_{j}\right)=\delta_{i j}$ pour $0 \leq j \leq i \leq 3$, contrairement à l'équation

$$
r g\left(E_{1}\right)^{2} \cdot r g\left(E_{0}\right)^{2} \cdot\left(\Delta_{1}\left(E_{1}\right)-\Delta_{1}\left(E_{0}\right)\right)^{2}=r g\left(E_{3}\right)^{2} \cdot r g\left(E_{2}\right)^{2} \cdot\left(\Delta_{1}\left(E_{3}\right)-\Delta_{1}\left(E_{2}\right)\right)^{2} .
$$

On remarquera d'ailleurs que dans le théorème $\mathrm{A}$, on a considéré les carrés des rangs et non les rangs des fibrés exceptionnels comme des variables.

2) Les théorèmes A,B,C sont aussi valables pour les bases d'hélices de fibrés exceptionnels sur $\mathbb{P}_{1} \times \mathbb{P}_{1}$. La variété $Z(\phi)$ qui intervient ici n'est pas isomorphe à $Z\left(\phi_{K}\right)$. On peut donner comme dans le cas de $\mathbb{P}_{3}$ une description géométrique de $Z(\phi)$, basée sur des hyperboles déjà utilisées dans [8] (pour plus de détails, voir [2]). Le sous-espace vectoriel de $H^{0}\left(\mathbb{P}_{2}, \mathcal{O}(2)\right.$ ) qui intervient ici est celui des équations de coniques passant par deux points fixes distincts de $\mathbb{P}_{2}$ 
(alors que dans le cas de $\mathbb{P}_{3}$ on utilisait les coniques passant par un point fixe et tangentes à une droite fixe de $\mathbb{P}_{2}$ en ce point).

3) On peut aussi étendre les théorèmes A,B,C aux surfaces de Hirzebruch et aux volumes de Fano considérés dans [7]. La similitude de tous ces cas vient du fait que le groupe de Grothendieck des variétés considérées est isomorphe à $\mathbb{Z}^{4}$.

4) Il est vraisemblable que les courbes planes associées aux fibrés exceptionnels sont utiles dans la description des invariants des faisceaux semi-stables sur $\mathbb{P}_{3}$. C'est vrai dans le cas de $\mathbb{P}_{1} \times \mathbb{P}_{1}$ (cf. [9]). Dans le cas de $\mathbb{P}_{2}$, ce qui intervient est un ensemble de points sur un droite (cf. [3]).

5) On pourrait, au lieu du plan $\Delta_{2}=\frac{1}{4}$, utiliser un plan d'équation $\Delta_{2}=c, c$ étant un scalaire quelconque. On obtiendrait le même type de résultat. Mais pour plusieurs raisons, le plan obtenu en prenant $c=\frac{1}{4}$ est le plus naturel. Par exemple, si $E$ est un faisceau semi-stable sur $\mathbb{P}_{3}$, l'inégalité $\Delta_{2}(E)>\frac{1}{4}$ est équivalente au fait que la dimension espérée de la variété de modules qui contient $E$ est strictement positive. On peut d'ailleurs conjecturer que c'est le cas si $E$ n'est pas une somme directe de fibrés exceptionnels.

6) Il est possible d'utiliser d'autres variétés que $Z\left(\phi_{K}\right)$ pour modéliser les bases d'hélices de fibrés exceptionnels. Par exemple, on peut prendre la sous-variété de $\mathbb{P}(W)^{4}$ constituée des $\left(l_{0}, l_{1}, l_{2}, l_{3}\right)$ tels que pour $1 \leq i \leq 3, l_{i}$ soit orthogonal aux $l_{j}, j<i$. Mais le choix de $Z\left(\phi_{K}\right)$ fait apparaître plus clairement les notions de paire exceptionnelle et de suite de fibrés exceptionnels associée, qui est essentielle dans la démonstration du théorème A.

7) Il est vraisemblable qu'on a des résultats analogues aux théorèmes A,B,C en ce qui concerne les fibrés exceptionnels sur $\mathbb{P}_{2}$ (avec des démonstrations plus simples).

Je tiens à remercier G.Trautmann pour de nombreuses discussions qui m'ont beaucoup aidé dans la réalisation de ce travail, ainsi que le DFG et l'Université de Kaiserslautern pour leur hospitalité.

\section{Plan des chapitres suivants :}

Dans le chapitre 1 on définit les invariants $\Delta_{i}$ et on rappelle la définition des hélices de fibrés exceptionnels.

Dans le chapitre 2 on définit et étudie les variétés $Z(\phi)$. Seuls les paragraphes 1 et 2 sont indispensables à la démonstration des théorèmes A,B,C.

Dans le chapitre 3 on définit les courbes et surfaces associées aux fibrés exceptionnels et on décrit la définition géométrique de $Z\left(\phi_{K}\right)$. Ce chapitre n'est pas nécessaire à la démonstration des théorèmes $\mathrm{A}, \mathrm{B}, \mathrm{C}$.

Dans le chapitre 4 on démontre les théorèmes A,B,C. 


\section{PrÉliminaires}

\subsection{Variables dérivées des classes de Chern}

Soient $X$ une variété algébrique projective lisse de dimension $n, E$ un fibré vectoriel de rang $r>0$ sur $X$. On définit des éléments $\Delta_{i}(E)$ de $A^{i}(E) \otimes \mathbb{Q}$, pour $1 \leq i \leq n$ formellement par

$$
\log (\operatorname{ch}(E))=\log (r)+\sum_{1 \leq i \leq n}(-1)^{i+1} \Delta_{i}(E)
$$

$c h(E)$ désignant le caractère de Chern de $E$. Un calcul simple montre qu'on a

$$
\begin{gathered}
\Delta_{1}(E)=\frac{c_{1}(E)}{r}, \Delta_{2}(E)=\frac{1}{r}\left(c_{2}(E)-\frac{r-1}{2 r} c_{1}(E)^{2}\right), \\
\Delta_{3}(E)=\frac{1}{r}\left(\frac{c_{3}(E)}{2}+c_{1}(E) c_{2}(E)\left(\frac{1}{r}-\frac{1}{2}\right)+c_{1}(E)^{3}\left(\frac{1}{3 r^{2}}-\frac{1}{2 r}+\frac{1}{6}\right)\right) .
\end{gathered}
$$

Proposition 1.1. Soient $L$ un fibré en droites, $E, F$, des fibrés vectoriels sur $X$. Alors on a 1) $\Delta_{1}(L)=c_{1}(L)$ et $\Delta_{i}(L)=0$ si $i>1$.

2) $\Delta_{i}(E \otimes F)=\Delta_{i}(E)+\Delta_{i}(F)$ si $1 \leq i \leq n$. Donc $\Delta_{i}(E \otimes L)=\Delta_{i}(E)$ si $2 \leq i \leq n$.

3) $\Delta_{i}\left(E^{*}\right)=(-1)^{i} \Delta_{i}(E)$ si $1 \leq i \leq n$.

Immédiat.

Il est clair que $\operatorname{ch}(E) / r$ est un polynôme en $\Delta_{1}(E), \ldots, \Delta_{n}(E)$. Il en découle que le théorème de Riemann-Roch s'écrit

$$
\frac{\chi(E)}{r}=P\left(\Delta_{1}(E), \ldots, \Delta_{n}(E)\right)
$$

$P$ étant un polynôme à coefficients rationnels ne dépendant que de $X$. Dans le cas où $X=\mathbb{P}_{3}$ on a

$$
P\left(\Delta_{1}, \Delta_{2}, \Delta_{3}\right)=\Delta_{3}-\Delta_{1} \Delta_{2}-2 \Delta_{2}+\frac{\left(\Delta_{1}+3\right)\left(\Delta_{1}+2\right)\left(\Delta_{1}+1\right)}{6} .
$$

\subsection{Fibrés exceptionnels et bases d'hélices sur $\mathbb{P}_{3}$ (cf. [5])}

Une hélice $\gamma=\left(E_{i}\right)_{i \in \mathbb{Z}}$ de fibrés exceptionnels possède les propriétés suivantes :

1) C'est une suite périodique, c'est-à-dire qu'on a $E_{i+4} \simeq E_{i}(4)$ pour tout $i \in \mathbb{Z}$.

2) On a $\chi\left(E_{i}, E_{j}\right)=0$ si $j<i<j+4$.

3) Pour tout entier i, le morphisme canonique

$$
e v: E_{i-1} \otimes \operatorname{Hom}\left(E_{i-1}, E_{i}\right) \longrightarrow E_{i} \quad\left(\operatorname{resp} . \quad e v^{*}: E_{i} \longrightarrow E_{i+1} \otimes \operatorname{Hom}\left(E_{i}, E_{i+1}\right)^{*}\right)
$$

est surjectif (resp. injectif) et son noyau (resp. conoyau) est un fibré exceptionnel $E$ (resp. $F)$. De plus,la suite périodique de fibrés vectoriels basée sur $\left(E_{i-2}, E, E_{i-1}, E_{i+1}\right)$ (resp. $\left(E_{i-1}, E_{i+1}, F, E_{i+2}\right)$ ) est une hélice (le terme $E$ (resp. $F$ ) étant d'indice $i-1$ (resp. $\left.i+1\right)$ ). 
Cette hélice s'appelle mutation à gauche (resp. mutation à droite) de $\gamma$ en $E_{i}$, et est notée $L_{E_{i}}(\gamma)$ (resp. $R_{E_{i}}(\gamma)$ ).

4) On pose $L_{E_{i}}^{2}=L_{E} \circ L_{E_{i}}(\gamma)$. C'est une hélice ayant pour base une suite de la forme $\left(E_{i-3}, E^{\prime}, E_{i-2}, E_{i-1}\right)$. On pose $L_{E_{i}}^{3}=L_{E^{\prime}} \circ L_{E_{i}}^{2}(\gamma)$. C'est une hélice ayant pour base une suite de la forme $\left(E^{\prime \prime}, E_{i-3}, E_{i-2}, E_{i-1}\right)$. Alors on a $E^{\prime \prime} \simeq E_{i-4}$, c'est-à-dire que $L_{E_{i}}^{3}(\gamma)=\gamma$.

5) On a $L_{E_{i}} \circ L_{E_{i+1}}^{2}(\gamma)=L_{E_{i+1}}^{2} \circ L_{E_{i}}(\gamma)$.

On a bien sûr des propriétés analogues à 4) et 5) concernant les mutations à droite. L'hélice la plus simple est $(\mathcal{O}(i))_{i \in \mathbb{Z}}$ et toute hélice de fibrés exceptionnels sur $\mathbb{P}_{3}$ peut s'obtenir en partant de cette hélice et en lui faisant subir une suite finie de mutations et une translation des indices (cf. [7]).

\section{LES VARIÉTÉS $Z(\phi)$}

\subsection{Définition de $Z(\phi)$}

Soit $W$ un $\mathbb{C}$-espace vectoriel de dimension 4 . Soient $\phi: W \longrightarrow W^{*}$ un isomorphisme linéaire, et $q$ la forme quadratique sur $W$ associée : $q(x)=x . \phi(x)$ pour tout $x \in W$. On supposera que $q \neq 0$, c'est-à-dire que $\phi$ n'est pas antisymétrique. On note $Q(\phi)$ la quadrique de $\mathbb{P}(W)$ des élément isotropes pour $q$. Le choix d'un élément non nul de $\wedge^{4} W^{*}$ définit un isomorphisme $j: \wedge^{2} W \longrightarrow \wedge^{2} W^{*}$. On pose $\xi_{0}(\phi)=j \circ \wedge^{2} \phi$, qui est un automorphisme de $\wedge^{2} W$ et induit un automorphisme $\xi(\phi)$ de la grassmannienne $G_{2,4}$ des plans de $W$. On a donc, pour tout plan $u$ de $W$

$$
\xi(\phi)(u)=\phi(u)^{\perp} .
$$

On supposera que $\wedge^{2} W$ est muni du produit scalaire déduit de $j$. En particulier, si $u, v$ sont des plans de $W$, on aura $u . v=0$ si et seulement si $u \cap v \neq \emptyset$. On pose $\nu(\phi)={ }^{t} \phi^{-1} \circ \phi$, qui est un élément de $G L(W)$. On a

$$
\xi(\phi) \circ \xi(\phi)=\wedge^{2} \nu(\phi) .
$$

On note $Z(\phi)$ la sous-variété fermée de $G_{2,4} \times G_{2,4}$ constituée des $\left(u, u^{\prime}\right)$ tels que

$$
u^{\prime} \cdot u=0 \quad \text { et } \quad u^{\prime} \cdot \xi(\phi)(u)=0 .
$$

Lemme 2.1. Si $\left(u, u^{\prime}\right)$ est un élement de $Z(\phi)$, il en est de même de $\left(u^{\prime}, \xi(\phi)(u)\right)$. Le morphisme

$$
\begin{gathered}
\tau(\phi): Z(\phi) \longrightarrow Z(\phi) \\
\left(u, u^{\prime}\right) \longmapsto\left(u^{\prime}, \xi(\phi)(u)\right)
\end{gathered}
$$

est un automorphisme de $Z(\phi)$. 
Immédiat .

Remarque : On peut montrer que la $G L(W)$-orbite de $\phi$ est entièrement déterminée par la connaissance de $\nu(\phi)$. Ceci implique que $Z(\phi)$ est entièrement déterminée si on connait $\nu(\phi)$ (cf. [2]).

\subsection{Quelques propriétés de $Z(\phi)$}

Proposition 2.2. La variété $Z(\phi)$ est irréductible.

Lemme 2.3. Le morphisme $\xi(\phi)$ possède un nombre fini de points fixes.

Démonstration. Soit $Y$ la sous-variété de $G_{2,4}$ des points fixes de $\xi(\phi)$. Si $u \in Y$, il est clair que $u \subset Q(\phi)$. Si $Y$ est de dimension au moins 2, $Q(\phi)$ est donc de rang 1 ou 2 , et il existe un hyperplan $H_{0}$ de $W$ tel que $Y$ contienne tous les plans de $W$ contenus dans $H_{0}$. Soient $u, v$ des points distincts de $Y$, avec $u \subset H_{0}, v \subset H_{0}$. On a alors

$$
H_{0}=u+v,
$$

donc

$$
\phi\left(H_{0}\right)=\phi(u+v)=\phi(u)+\phi(v)=u^{\perp}+v^{\perp}=(u \cap v)^{\perp} .
$$

Donc $u \cap v=\phi\left(H_{0}\right)^{\perp}$, et $Y$ ne contient donc pas tous les plans contenus dans $H_{0}$. Donc $\operatorname{dim}(Y) \leq 1$.

Notons $U$ le sous-fibré universel de $W \otimes \mathcal{O}_{G_{2,4}}$. Soit

$$
P(\phi)=\mathbb{P}(U) \times_{G_{2,4}} \mathbb{P}\left(\xi(\phi)^{*} U\right) .
$$

C'est une variété irréductible. On note $\pi$ la projection $P(\phi) \longrightarrow G_{2,4}$. Soient

$$
\begin{gathered}
C=\{(u, v) \in Z(\phi), u=v\}, \quad C^{\prime}=\{(u, v) \in Z(\phi), \xi(\phi)(u)=v\}, \\
B=\{(u, v) \in Z(\phi), u \cap \xi(\phi)(u) \cap v \neq\{0\}\} .
\end{gathered}
$$

Ce sont des sous-variétés fermées de $Z(\phi)$.

Soient $V$ la sous-variété fermée de $G_{2,4}$ constituée des $u$ tels que $u . \xi(\phi)(u)=0$ et

$$
\begin{gathered}
W_{0}=\left\{\left(l_{1}, l_{2}\right) \in P(\phi), l_{1}=l_{2}\right\}, \\
W_{0}^{\prime}=\left\{\left(l_{1}, l_{2}\right) \in P(\phi), l_{1} \text { ou } l_{2} \subset \pi\left(l_{1}, l_{2}\right) \cap \xi(\phi)\left(\pi\left(l_{1}, l_{2}\right)\right)\right\},
\end{gathered}
$$

qui sont des sous-variétés fermées de $P(\phi)$ au dessus de $V$.

Lemme 2.4. La variété $V$ est une hypersurface de $G_{2,4}$ qui est irréductible si $Q(\phi)$ l'est.

Démonstration. Soient $u$ un point de $V$ et $l_{1}$ une droite de $u \cap \xi(\phi)(u)$. Alors $l_{1}$ est un point de $Q(\phi)$ et $u \subset{ }^{t} \phi\left(l_{1}\right)^{\perp}$. Réciproquement, si $l_{1}$ est un point de $Q(\phi)$, tout plan de $W$ contenant $l_{1}$ et contenu dans ${ }^{t} \phi\left(l_{1}\right)^{\perp}$ est un point de $V$. On a donc un morphisme surjectif $\mathbb{P}(E) \longrightarrow V$, $E$ désignant le fibré vectoriel de rang 2 sur $Q(\phi)$ défini par : $E_{l}={ }^{t} \phi(l) / l$ pour tout $l \in Q(\phi)$. Il en découle que $V$ est de dimension 3 , donc est une hypersurface de $G_{2,4}$, et est irréductible si $Q(\phi)$ l'est. 
Démontrons maintenant la proposition 2.2. Il découle aisément des deux lemmes précédents que $W_{0}$ est de dimension 3 et irréductible si $Q(\phi)$ l'est, et que $W_{0}^{\prime}$ est de dimension 4.

Considérons les morphismes rationnels

$$
\begin{gathered}
f: Z(\phi) \longrightarrow P(\phi) \\
(u, v) \longmapsto(u \cap v, \xi(\phi)(u) \cap v),
\end{gathered}
$$

et

$$
\begin{aligned}
g & : P(\phi) \longrightarrow Z(\phi) \\
\left(l_{1}, l_{2}\right) & \longmapsto\left(\pi\left(l_{1}, l_{2}\right), l_{1} \oplus l_{2}\right) .
\end{aligned}
$$

Le morphisme $f$ est régulier au dessus de $Z(\phi) \backslash\left(C \cup C^{\prime}\right)$, et $g$ l'est au dessus de $P(\phi) \backslash W_{0}$. Le morphisme $f$ induit un isomorphisme

$$
Z(\phi) \backslash B \simeq P(\phi) \backslash\left(W_{0} \cup W_{0}^{\prime}\right)
$$

dont l'inverse est la restriction de $g$.

D'autre part, soit $\pi_{1}: Z(\phi) \longrightarrow G_{2,4}$ la première projection. Si $u$ est un point de $G_{2,4} \backslash V$, on a

$$
\pi_{1}^{-1}(u) \simeq \mathbb{P}(u) \times \mathbb{P}(\xi(\phi)(u)) \simeq \mathbb{P}_{1} \times \mathbb{P}_{1}
$$

Si u est un point de $V$ qui n'est pas un point fixe de $\xi(\phi), \pi_{1}^{-1}(u)$ est constitué des deux plans projectifs distincts : $P_{1}(u)=\{(u, v), v \subset u+\xi(\phi)(u)\}$ et $P_{2}(u)=\{(u, v), u \cap \xi(\phi)(u) \subset v\}$. Enfin, si $\xi(\phi)(u)=u, \pi_{1}^{-1}(u)$ est de dimension 3. La variété $B$ est constituée des plans $P_{2}(u)$ ci-dessus, $u$ parcourant l'ouvert de $V$ des points non invariants par $\xi(\phi)$, et des fibres de $\pi_{1}$ au dessus des points fixes de $\xi(\phi)$. Il en découle que $B$ est de dimension 5 .

Maintenant, les composantes irréductibles de $Z(\phi)$ sont de dimension au moins 6 , car $Z(\phi)$ est une sous-variété de $G_{2,4} \times G_{2,4}$ définie par deux équations. Donc pour montrer que $Z(\phi)$ est irréductible il suffit de montrer que $Z(\phi) \backslash B$ l'est, ce qui découle du fait que $Z(\phi) \backslash B \simeq P(\phi) \backslash\left(W_{0} \cup W_{0}^{\prime}\right)$ et que $P(\phi)$ est irréductible.

Remarques : 1) Soit $S$ la sous-variété fermée de $Z(\phi)$ constituée des points $(u, v)$ tels qu'il existe une droite $l$ de $W$ telle que $l \subset u, v \subset \phi(l)^{\perp}$. Rappelons que

$$
C=\{(u, v) \in Z(\phi), u=v\}, \quad C^{\prime}=\{(u, v) \in Z(\phi), \xi(\phi)(u)=v\} .
$$

Alors on peut montrer que $Z(\phi)$ est normale et que l'ensemble des points singuliers de $Z(\phi)$ est $C \cup C^{\prime} \cup S$ (cf. [2], prop. 2.9).

2) On suppose que $Q(\phi)$ est intègre. Soient $p_{1}, p_{2}$ les projections $Z(\phi) \longrightarrow G_{2,4}, D$ une section hyperplane de $G_{2,4}, \Gamma$ un hyperplan de $\mathbb{P}(W)$,

$$
\begin{gathered}
P=p_{1}^{*}(D), \quad P^{\prime}=p_{1}^{*}(D), \\
K_{1}=\{(u, v) \in Z(\phi), u \cap v \cap \Gamma \neq \emptyset\}, K_{2}=\{(u, v) \in Z(\phi), \xi(\phi)(u) \cap v \cap \Gamma \neq \emptyset\} .
\end{gathered}
$$

On montre alors que $P, P^{\prime}, K_{1}$ et $K_{2}$ sont des hypersurfaces de $Z(\phi)$ et que le groupe des classes d'équivalence linéaire de diviseurs de Weil de $Z(\phi)$ est isomorphe à $\mathbb{Z}^{4}$, et est engendré par $P, P^{\prime}, K_{1}$ et $K_{2}$ (cf. [2], thm. 2.11). 


\section{3 - Interprétation géométrique}

On suppose que $W$ est un sous-espace vectoriel de $H^{0}\left(\mathbb{P}_{2}, \mathcal{O}(2)\right)$. Soit

$$
\sigma: \mathbb{P}_{2} \longrightarrow \mathbb{P}(W)
$$

un morphisme rationnel. On suppose que :

1) $\sigma^{*}(\mathcal{O}(1))=\mathcal{O}(2)$.

2) $\sigma^{*}: W^{*} \longrightarrow H^{0}\left(\mathbb{P}_{2}, \mathcal{O}(2)\right)$ induit un isomorphisme $W^{*} \simeq W$.

3) Pour tout point $P$ de $\mathbb{P}_{2}$ où $\sigma$ est défini, on a $P \in \sigma(P), \sigma(P)$ étant vu comme une conique de $\mathbb{P}_{2}$.

On note $\phi$ l'inverse de $\sigma^{*}: W^{*} \longrightarrow W$.

Lemme 2.5. Le morphisme $\sigma$ est à valeurs dans $Q(\phi)$, et pour tous points $P, Q$ de $\mathbb{P}_{2}$ où $\sigma$ est défini, on a $Q \in \sigma(P)$ si et seulement si $\phi(\sigma(P)) . \sigma(Q)=0$.

Démonstration. La seconde assertion découle du fait qu'on a

$$
\phi(\sigma(P)) \cdot \sigma(Q)=\sigma^{*} \circ \phi(\sigma(P))(Q)=\sigma(P)(Q) .
$$

La première en découle car si $\sigma$ est défini en $P$ on a

$$
\phi(\sigma(P))(\sigma(P))=\sigma(P)(P)=0
$$

d'après la propriété 3 de $\sigma$, et donc $\sigma(P) \in Q(\phi)$.

Proposition 2.6. Si $\sigma$ vérifie les propriétés 1,2,3, on est dans l'un des trois cas suivants :

1) $\mathbb{P}(W)$ est l'espace des coniques passant par deux points fixes distincts de $\mathbb{P}_{2}$.

2) $\mathbb{P}(W)$ est l'espace des coniques passant par un point fixe de $\mathbb{P}_{2}$ et tangentes à une droite fixe de $\mathbb{P}_{2}$ en ce point.

$\mathbb{P}(W)$ est l'espace des coniques invariantes par une involution non triviale de $\mathbb{P}_{2}$.

Dans les cas 1 et $2, \sigma$ induit un isomorphisme birationnel $\mathbb{P}_{2} \simeq Q(\phi)$.

Démonstration. Posons $V=H^{0}\left(\mathbb{P}_{2}, \mathcal{O}(1)\right)^{*}$, et soit $(x, y, z)$ une base de $V$. Moyennant un changement de base de $V^{*}$, on peut supposer que $W^{\perp} \subset S^{2} V$ admet pour base un des 7 couples suivants :

$$
\left(x^{2}, x y\right),\left(x^{2}, y^{2}\right),(x y, x z),(x y, z(x+y+z)),(x y, z(x+y)),(x y, z(x+z)),\left(x y, z^{2}\right) .
$$

Les trois premiers ces sont ceux de la proposition. Il faut montrer que les autres cas ne peuvent pas se produire. Posons

$$
\phi^{-1}=\sum_{0 \leq i \leq 3} s_{i} \otimes t_{i},
$$

avec $s_{i}, t_{i} \in W$. La condition 3 sur $\sigma$ signifie que $\sum_{0 \leq i \leq 3} s_{i} . t_{i}=0$ dans $H^{0}\left(\mathbb{P}_{2}, \mathcal{O}(4)\right)$. Cela implique que la restriction à $S^{2} W$ du morphisme canonique

$$
S^{2}\left(H^{0}\left(\mathbb{P}_{2}, \mathcal{O}(2)\right) \longrightarrow H^{0}\left(\mathbb{P}_{2}, \mathcal{O}(4)\right)\right.
$$


n'est pas injective. L'examen des cas 1 à 7 montre que seuls les cas 1,2,3 sont possibles.

Montrons que $\sigma$ induit un isomorphisme $\mathbb{P}_{2} \simeq Q(\phi)$ dans le cas 2 (l'autre cas est analogue). Relativement à une base convenable de $W$, il est clair que $\sigma$ est de la forme

$$
(a, b, c) \longmapsto\left(a c, b^{2}, b c, c^{2}\right) .
$$

Donc $\sigma$ est un isomorphisme birationnel de $\mathbb{P}_{2}$ sur une quadrique irréductible. Puisque d'après le lemme 2.5, $\sigma$ est à valeurs dans $Q(\phi)$, il reste à voir que ce dernier n'est pas $\mathbb{P}(W)$ tout entier, c'est-à-dire que $\phi$ n'est pas antisymétrique. Soit $(X, Y, Z)$ la base de $V^{*}$ duale de $(x, y, z)$. D'après les propriétés de $\sigma$, on voit que si $W$ est muni de la base $\left(X Z, Y^{2}, Y Z, Z^{2}\right)$, la matrice de ${ }^{t} \phi^{-1}$ relativement à cette base et à la base duale est de la forme

$$
\left(\begin{array}{cccc}
0 & 0 & c_{0} & d_{0} \\
-c_{0} & 0 & c_{1} & d_{1} \\
-d_{0} & -c_{1} & c_{2} & d_{2} \\
0 & -d_{1}-c_{2} & -d_{2} & 0
\end{array}\right)
$$

Il est donc clair que $\phi$, étant un isomorphisme, ne peut pas être antisymétrique.

On suppose qu'on est dans le cas 1 ou le cas 2 de la proposition 2.6. Si $P_{0}, P_{1}$ des points de $\mathbb{P}_{2}$, on note $C\left(P_{0}, P_{1}\right)$ le sous-espace vectoriel de $W$ constitué des coniques passant par $P_{0}$ et $P_{1}$. Si $P_{0}$ et $P_{1}$ sont généraux, $C\left(P_{0}, P_{1}\right)$ est un plan de $W$.

Proposition 2.7. Soient $P_{0}, P_{1}$ des points généraux de $\mathbb{P}_{2}$. Alors $\sigma\left(P_{0}\right) \cap \sigma\left(P_{1}\right)$ contient deux points distincts $P_{2}, P_{3}$ en plus des points de base de $W$, et on a

$$
\xi(\phi)\left(\sigma\left(P_{0}\right) \wedge \sigma\left(P_{1}\right)\right)=\sigma\left(P_{2}\right) \wedge \sigma\left(P_{3}\right), \xi(\phi)\left(C\left(P_{0}, P_{1}\right)\right)=C\left(P_{2}, P_{3}\right)=\sigma\left(P_{0}\right) \wedge \sigma\left(P_{1}\right) .
$$

Démonstration. Cela découle du lemme 2.5.

Remarques : 1) On suppose qu'on est dans le cas 1 ou le cas 2 de la proposition 2.6. Alors $T=\sigma^{-1} \circ \nu(\phi) \circ \sigma$ est un automorphisme birationnel de $\mathbb{P}_{2}$, et pour tout point $P$ de $\mathbb{P}_{2}$ où $\sigma$ est défini, $\sigma$ est aussi défini en $T(P)$ et on a $T(P) \in \sigma(P)$. On a $\sigma \circ T=T \circ \sigma$. Dans la situation de la proposition 2.7, les points de $\sigma\left(P_{2}\right) \cap \sigma\left(P_{3}\right)$ autres que les points de base de $W$ sont $T\left(P_{0}\right)$ et $T\left(P_{1}\right)$.

2) Soient $W_{0}$ un $\mathbb{C}$-espace vectoriel de dimension $4, \phi: W_{0} \longrightarrow W_{0}^{*}$ un isomorphisme tel que $Q(\phi)$ soit une quadrique de rang au moins 3. Alors on peut montrer qu'il existe un morphisme $\sigma$ possédant les propriétés 1,2,3 et un isomorphisme $\epsilon: W \longrightarrow W_{0}$ tels que $\sigma^{*}=\epsilon \circ \phi^{-1} \circ{ }^{t} \epsilon$. Cependant deux tels $\sigma$ ne peuvent pas en général se déduire l'un de l'autre par un automorphisme de $\mathbb{P}_{2}$.

3) Géométriquement parlant, un point de $Z(\phi)$ est un couple

$$
\omega=\left(\sigma\left(P_{0}\right) \wedge \sigma\left(P_{1}\right), \sigma\left(Q_{0}\right) \wedge \sigma\left(Q_{1}\right)\right)
$$

tel qu'il existe une conique élément de $\mathbb{P}(W)$ passant par $P_{0}, P_{1}, Q_{0}, Q_{1}$, ainsi qu'une conique élément de $\mathbb{P}(W)$ passant par $P_{2}, P_{3}, Q_{0}, Q_{1}$, où $P_{2}, P_{3}$ sont les points d'inter-section de $\sigma\left(P_{0}\right)$ et $\sigma\left(P_{1}\right)$ autres que les points de base de $W$. Le lemme 2.1 signifie que l' on a

$$
\tau(\phi)(\omega)=\left(\sigma\left(Q_{0}\right) \wedge \sigma\left(Q_{1}\right), \sigma\left(P_{2}\right) \wedge \sigma\left(P_{3}\right)\right) .
$$


Les points de $\sigma\left(P_{2}\right) \cap \sigma\left(P_{3}\right)$ autres que les points de base de $W$ sont $T\left(P_{0}\right)$ et $T\left(P_{1}\right)$. On a

$$
\tau(\phi)^{4}(\omega)=\left(T\left(\sigma\left(P_{0}\right)\right) \wedge T\left(\sigma\left(P_{1}\right)\right), T\left(\sigma\left(Q_{0}\right)\right) \wedge T\left(\sigma\left(Q_{1}\right)\right)\right) .
$$

\section{Exemples :}

1) Un exemple du cas 1 est fourni par l'étude des fibrés exceptionnels sur $\mathbb{P}_{1} \times \mathbb{P}_{1}$ (cf. [8]). Dans ce cas $W$ est engendré par $X Y, X Z, Y Z$ et $Z^{2}$, et $\sigma$ est dans cette base le morphisme

$$
(x, y, z) \longmapsto\left(z^{2}, z^{2}-y z, z^{2}-x z, x y-x z-y z\right),
$$

c'est-à-dire que $\sigma$ associe au point $(x, y)$ de $\mathbb{R}^{2}$ l'hyperbole d'équation

$$
(X-(x-1))(Y-(y-1))=1 \text {. }
$$

On dans ce cas $T(x, y, z)=(x-2 z, y-2 z, z)$ et $T$ est, restreint à $\mathbb{R}^{2}$, la translation de vecteur $(-2,-2)$.

2) Dans le cas 2, un exemple de morphisme $\sigma$ sera donné au chapitre 3, pour lequel l'automorphisme $T$ de $\mathbb{P}_{2}$ n'est pas linéaire. Mais il est possible aussi dans le cas 2 que $T$ soit linéaire. Par exemple, supposons que $W$ soit engendré par $X Z, Y^{2}, Y Z$ et $Z^{2}$. On considère le morphisme $\sigma$ donné par :

$$
\sigma(x, y, z)=\left(y z,-\frac{z^{2}}{2}, y z-x z,-\frac{y^{2}}{2}\right) .
$$

Dans ce cas on a $T(x, y, z)=(x-2 y, y,-z)$.

\subsection{Mutations}

On se place sous les hypothèses du $\S 2.1$.

Lemme 2.8. Soit $(u, v)$ un point de $Z(\phi)$.

1) On suppose que $u \neq v$ et $u \cap v \neq \xi(\phi)(u) \cap \xi(\phi)(v)$. Alors

$$
(v,(u \cap v) \oplus(\xi(\phi)(u) \cap \xi(\phi)(v)))
$$

est aussi un élément de $Z(\phi)$.

2) On suppose que $\xi(\phi)(u) \neq v$ et $\xi(\phi)(u) \cap v \neq \xi(\phi)(v) \cap \nu(\phi)(u)$. Alors

$$
(v,(\xi(\phi)(u) \cap v) \oplus(\xi(\phi)(v) \cap \nu(\phi)(u)))
$$

est aussi un élément de $Z(\phi)$.

Immédiat.

On a donc deux morphismes rationnels $L_{1}$ et $L_{2}$, de $Z(\phi)$ dans lui-même, appelés mutations et définis par :

$$
\begin{gathered}
L_{1}(u, v)=\left(\xi(\phi)^{-1}((u \cap v) \oplus(\xi(\phi)(u) \cap \xi(\phi)(v))), v\right), \\
L_{2}(u, v)=\left(\xi(\phi)^{-1}((\xi(\phi)(u) \cap v) \oplus(\xi(\phi)(v) \cap \nu(\phi)(u))), v\right) .
\end{gathered}
$$


Proposition 2.9. On a

$$
L_{2}=\tau(\phi) \circ L_{1} \circ \tau(\phi)^{-1} \circ L_{1} \circ \tau(\phi)
$$

et

$$
L_{1}=\tau(\phi)^{-1} \circ L_{2} \circ \tau(\phi) \circ L_{2} \circ \tau(\phi)^{-1}
$$

Démonstration. On ne démontrera que la première égalité, la seconde étant analogue. Soit $(u, v)$ un point général de $Z(\phi)$. Posons

$$
\begin{gathered}
u_{0}=\xi(\phi)^{-1}((v \cap \xi(\phi)(u)) \oplus(\xi(\phi)(v) \cap \nu(\phi)(u))), \\
u_{1}=\xi(\phi)^{-1}\left(\left(u \cap u_{0}\right) \oplus\left(\xi(\phi)(u) \cap \xi(\phi)\left(u_{0}\right)\right)\right) .
\end{gathered}
$$

On a

$$
\begin{aligned}
\tau(\phi) \circ L_{1} \circ \tau(\phi)^{-1} \circ L_{1} \circ \tau(\phi)(u, v) & =\tau(\phi) \circ L_{1} \circ \tau(\phi)^{-1} \circ L_{1}(v, \xi(\phi)(u)) \\
& =\tau(\phi) \circ L_{1} \circ \tau(\phi)^{-1}\left(u_{0}, \xi(\phi)(u)\right), \\
& =\tau(\phi) \circ L_{1}\left(u, u_{0}\right), \\
& =\tau(\phi)\left(u_{1}, u_{0}\right)=\left(u_{0}, \xi(\phi)\left(u_{1}\right)\right) .
\end{aligned}
$$

Il faut donc montrer que $\xi(\phi)\left(u_{1}\right)=v$. Pour cela on remarque d'abord que $\xi(\phi)\left(u_{1}\right) \cap \xi(\phi)\left(u_{0}\right)$ contient $\xi(\phi)(u) \cap \xi(\phi)\left(u_{0}\right)$, qui contient lui-même $v \cap \xi(\phi)(u)$. Il reste à démontrer que $u \cap u_{0} \subset v$. En effet, si cela est démontré on aura $v=\left(u \cap u_{0}\right) \oplus(v \cap \xi(\phi)(u))=\xi(\phi)\left(u_{1}\right)$. L'inclusion $u \cap u_{0} \subset v$ équivaut à $\xi(\phi)(v) \subset \xi(\phi)(u)+\xi(\phi)\left(u_{0}\right)$, ce qui est vrai car $\xi(\phi)(v) \cap \xi(\phi)(u) \neq\{0\}$ et $\xi(\phi)(v) \cap \xi(\phi)\left(u_{0}\right)=\xi(\phi)(v) \cap \nu(\phi)(u) \neq\{0\}$.

Proposition 2.10. Les morphismes $L_{1}, L_{2}$ sont des isomorphismes birationnels et on a

$$
\begin{aligned}
L_{1}^{-1}(u, v) & =\left(\left(\xi(\phi)^{-1}(v) \cap u\right) \oplus(v \cap \xi(\phi)(u)), v\right), \\
L_{2}^{-1}(u, v) & =\left(\left(\xi(\phi)^{-1}(u) \cap \xi(\phi)^{-1}(v)\right) \oplus(u \cap v), v\right) .
\end{aligned}
$$

Démonstration. Analogue à celle de la proposition 2.9 .

Proposition 2.11. On a

$$
\tau(\phi)^{-1} \circ L_{1} \circ \tau(\phi)^{3} \circ L_{1} \circ \tau(\phi)^{-1} \circ L_{1}=\tau(\phi) \circ L_{2} \circ \tau(\phi)^{-3} \circ L_{2} \circ \tau(\phi) \circ L_{2}=I_{Z(\phi)} .
$$

Immédiat.

Ce résultat généralise la propriété 4 des hélices de fibrés exceptionnels donnée au $\S 1.2$.

Proposition 2.12. On a $L_{1} \circ L_{2}=L_{1} \circ L_{2}$.

Démonstration. Analogue à celle de la proposition 2.9. 
Ce résultat correspond à la propriété 5 des hélices de fibrés exceptionnels donnée au $§ 1.2$.

Interprétation géométrique : on suppose que $\phi$ provient d'un morphisme rationnel

$\sigma: \mathbb{P}_{2} \longrightarrow \mathbb{P}(W)$ possédant les propriétés $1,2,3$ du $\S 2.1$. On suppose qu'on est dans le cas 1 ou le 2 de la proposition 2.6. Soient $P_{0}, P_{1}, Q_{0}, Q_{1}$ des points généraux de $\mathbb{P}_{2}$. Soient $P_{2}, P_{3}$ (resp. $\left.Q_{2}, Q_{3}\right)$ les points d'intersection de $\sigma\left(P_{0}\right)$ et $\sigma\left(P_{1}\right)$ (resp. $\sigma\left(Q_{0}\right)$ et $\sigma\left(Q_{1}\right)$ ) autres que les points de base de $W$. Alors il passe une unique conique $c$ (resp. $c^{\prime}$ ) élément de $\mathbb{P}(W)$ par $P_{0}, P_{1}, Q_{0}, Q_{1}$ (resp. $\left.P_{2}, P_{3}, Q_{2}, Q_{3}\right)$, et on a

$$
\tau(\phi) \circ L_{1}\left(\sigma\left(P_{0}\right) \wedge \sigma\left(P_{1}\right), \sigma\left(Q_{0}\right) \wedge \sigma\left(Q_{1}\right)\right)=\left(\sigma\left(Q_{0}\right) \wedge \sigma\left(Q_{1}\right), c \oplus c^{\prime}\right) .
$$

\subsection{La variété $Z(\phi)$ associée à $\mathbb{P}_{3}$}

Soient $W_{K}=K\left(\mathbb{P}_{3}\right) \otimes \mathbb{C}$ qu'on munit de la forme bilinéaire $\chi(x, y)$ telle que pour tous fibrés vectoriels $E, F$ sur $\mathbb{P}_{3}$ on ait $\chi([E],[F])=\chi\left(E^{*} \otimes F\right), \phi_{K}$ l'isomorphisme $W_{K} \longrightarrow W_{K}^{*}$ défini par $\chi(x, y)=x . \phi_{K}(y)$ pour tous $x, y$ dans $W_{K}$. Relativement à la base

$$
f_{0}=[\mathcal{O}(-4)], f_{1}=\left[\left(\wedge_{2} Q^{*}\right)(-3)\right], f_{2}=\left[Q^{*}(-3)\right], f_{3}=[\mathcal{O}(-3)],
$$

la matrice de $\phi_{K}$ est

$$
\left(\begin{array}{llll}
1 & 4 & 6 & 4 \\
0 & 1 & 4 & 6 \\
0 & 0 & 1 & 4 \\
0 & 0 & 0 & 1
\end{array}\right)
$$

Soit $\left(E_{0}, E_{1}, E_{2}, E_{3}\right)$ une base d'hélice de fibrés exceptionnels. Alors il est aisé de voir que $\left(\mathbb{C}\left[E_{0}\right] \oplus \mathbb{C}\right.$. $\left[E_{1}\right], \mathbb{C} .\left[E_{1}\right] \oplus \mathbb{C}$. $\left.\left[E_{2}\right]\right)$ est un élément de $Z\left(\phi_{K}\right)$. On obtient ainsi une application

$$
\Lambda_{K}: B\left(\mathbb{P}_{3}\right) \longrightarrow Z\left(\phi_{K}\right) \text {. }
$$

Soient $\tau$ et $L$ les automorphismes de $B\left(\mathbb{P}_{3}\right)$ définis par

$$
\tau\left(E_{0}, E_{1}, E_{2}, E_{3}\right)=\left(E_{1}, E_{2}, E_{3}, E_{0}(4)\right), L\left(E_{0}, E_{1}, E_{2}, E_{3}\right)=\left(E_{0}, E, E_{1}, E_{3}\right),
$$

$E$ étant le noyau du morphisme d'évaluation $E_{1} \otimes \operatorname{Hom}\left(E_{1}, E_{2}\right) \longrightarrow E_{2}$. Alors on a $\tau\left(\phi_{K}\right) \circ \Lambda_{K}=\Lambda_{K} \circ \tau$ et $L_{1} \circ \Lambda_{K}=\Lambda_{K} \circ L$. 


\section{Courbes Planes ASSOCIÉES AUX BASES D'HÉLICES DE FIBrÉS EXCEPTIONNELS}

\subsection{Courbes planes associées aux fibrés exceptionnels}

Soit $E$ un fibré exceptionnel de rang $r$ sur $\mathbb{P}_{3}$. On a alors $\chi\left(E^{*} \otimes E\right)=1$, d'où on déduit, compte tenu du théorème de Riemann-Roch tel qu'il est exprimé au $\S 1.1$ le

Lemme 3.1. On a $\Delta_{2}(E)=\frac{1}{4}\left(1-\frac{1}{r^{2}}\right)$.

Si maintenant $X$ est un fibré vectoriel sur $\mathbb{P}_{3}$, et si on pose $\Delta_{i}=\Delta_{i}(X)$ pour $1 \leq i \leq 3$, on a

$$
\frac{\chi\left(X^{*} \otimes E\right)}{r . r g(X)}=-\frac{1}{6} z^{3}+\left(\Delta_{2}+\Delta_{2}(E)+\frac{1}{6}\right) z+\Delta_{3}(E)-\Delta_{3},
$$

avec $z=\Delta_{1}-\Delta_{1}(E)-2$. Au fibré exceptionnel $E$ on associe la surface $S(E)$ de $\mathbb{R}^{3}$ (muni des coordonnées $\left.\Delta_{1}, \Delta_{2}, \Delta_{3}\right)$ d'équation

$$
\Delta_{3}=-\frac{1}{6}\left(\Delta_{1}-\Delta_{1}(E)-2\right)^{3}+\left(\Delta_{2}+\Delta_{2}(E)+\frac{1}{6}\right)\left(\Delta_{1}-\Delta_{1}(E)-2\right)+\Delta_{3}(E) .
$$

On note $P(E)$ le point de $\mathbb{R}^{3}$ correspondant à $E$, c'est-à-dire

$$
P(E)=\left(\Delta_{1}(E), \Delta_{2}(E), \Delta_{2}(E)\right),
$$

qui est d'après le lemme 3.1 situé en dessous du plan $H$ d'équation $\Delta_{2}=\frac{1}{4}$. Ce plan $H$ est muni des coordonnées $\Delta_{1}, \Delta_{3}$. On note $C(E)$ l'intersection de $H$ et $S(E)$. D'après le lemme 3.1, c'est la cubique d'équation

$$
\Delta_{3}=-\frac{1}{6}\left(\Delta_{1}-\Delta_{1}(E)-2\right)^{3}+\left(\frac{2}{3}-\frac{1}{4 r^{2}}\right)\left(\Delta_{1}-\Delta_{1}(E)-2\right)+\Delta_{3}(E) .
$$

\subsection{Paires exceptionnelles}

Soient $(E, F)$ une paire exceptionnelle, $\left(F_{i}\right)_{i \in Z}$ la suite de fibrés exceptionnels associée, définie par :

$$
F_{0}=E, F_{1}=F
$$

et pour tout entier $i$ on a une suite éxacte

$$
0 \longrightarrow F_{i-1} \longrightarrow F_{i} \otimes \operatorname{Hom}\left(F_{i}, F_{i+1}\right) \longrightarrow F_{i+1} \longrightarrow 0,
$$

dont le second morphisme est l'évaluation. On pose $k=\operatorname{dim}\left(\operatorname{Hom}\left(F_{i}, F_{i+1}\right)\right)$, qui est indépendant de $i$.

Proposition 3.2. La suite $\left(P\left(F_{i}\right)\right)_{i \in \mathbb{Z}}$ a des limites quand $i$ tend vers $+\infty$ ou $-\infty$. Ce sont des points de $H$. 
Démonstration. On a, pour tout entier $i$

$$
\Delta_{1}\left(F_{i+2}\right)=\frac{c_{1}\left(F_{i+2}\right)}{r g\left(F_{i+2}\right)}=\frac{k \cdot c_{1}\left(F_{i+1}\right)-c_{1}\left(F_{i}\right)}{k \cdot r g\left(F_{i+1}\right)-r g\left(F_{i}\right)},
$$

et le fait que $\lim _{i \rightarrow+\infty} \Delta_{1}\left(F_{i}\right)$ et $\lim _{i \rightarrow-\infty} \Delta_{1}\left(F_{i}\right)$ existent est une propriété bien connue des suites récurrentes. D'autre part, d'après le lemme 3.1 et le fait que $\lim _{i \rightarrow \pm \infty} r g\left(F_{i}\right)=+\infty$, on a $\lim _{i \rightarrow \pm \infty} \Delta_{2}\left(F_{i}\right)=\frac{1}{4}$, ce qui montre que les limites de $P\left(F_{i}\right)$ lorsque $i$ tend vers $+\infty$ ou $-\infty$, si elles existent, sont des points de $H$.

Il reste à voir que $\Delta_{3}\left(F_{i}\right)$ possède des limites lorsque $i$ tend vers $+\infty$ ou $-\infty$. Soient $E_{2}, E_{3}$ des fibrés exceptionnels tels que $\left(E, F, E_{2}, E_{3}\right)$ soit une base d'hélice. Alors pour tout entier $i$ il en est de même de $\left(F_{i}, F_{i+1}, E_{2}, E_{3}\right)$. On a donc $\chi\left(E_{2}^{*} \otimes F_{i}\right)=0$, et en utilisant la formule de Riemann-Roch donnée au $\S 1.1$, on obtient une équation du type

$$
\Delta_{3}\left(F_{i}\right)=\Phi\left(\Delta_{1}\left(F_{i}\right), \Delta_{2}\left(F_{i}\right)\right)
$$

$\Phi$ étant un polynôme indépendant de $i$. Il en découle, par continuité de $\Phi$, que les limites de $\Delta_{3}\left(F_{i}\right)$ lorsque $i$ tend vers $+\infty$ ou $-\infty$ existent.

Posons

$$
\Delta_{k+}=\lim _{i \rightarrow+\infty} \Delta_{k}\left(F_{i}\right), \quad \Delta_{k-}=\lim _{i \rightarrow-\infty} \Delta_{k}\left(F_{i}\right), k=1,3 .
$$

Les cubiques $C\left(F_{i}\right)$ ont des limites lorsque $i$ tend vers $+\infty$ ou $-\infty$. L'équation de $C_{+}=\lim _{i \rightarrow+\infty} C\left(F_{i}\right) \quad\left(\operatorname{resp} . C_{-}=\lim _{i \rightarrow-\infty} C\left(F_{i}\right)\right)$ est

$$
\begin{gathered}
\Delta_{3}=-\frac{1}{6}\left(\Delta_{1}-\Delta_{1+}-2\right)^{3}+\frac{2}{3}\left(\Delta_{1}-\Delta_{1+}-2\right)+\Delta_{3+} \\
\left(\text { resp. } \Delta_{3}=-\frac{1}{6}\left(\Delta_{1}-\Delta_{1-}-2\right)^{3}+\frac{2}{3}\left(\Delta_{1}-\Delta_{1-}-2\right)+\Delta_{3-}\right) .
\end{gathered}
$$

Ce sont des translatées de la cubique d'équation $\Delta_{3}=-\frac{1}{6} \Delta_{1}^{3}+\frac{2}{3} \Delta_{1}$. On a

$$
\left(\Delta_{1+}, \Delta_{3+}\right) \in C_{+},\left(\Delta_{1-}, \Delta_{3-}\right) \in C_{-} .
$$

\subsection{Construction géométrique de la variété $Z(\phi)$ associée à $\mathbb{P}_{3}$}

Les cubiques associées aux fibrés exceptionnels et aux paires exceptionnelles ont des équations du type

$$
\Delta_{3}=-\frac{1}{6}\left(\Delta_{1}-X_{0}\right)^{3}+\beta\left(\Delta_{1}-X_{0}\right)+Y_{0}
$$

Les cubiques vérifiant la condition $\beta=\frac{2}{3}$ sont dites spéciales. Dans ce cas le point $\left(X_{0}-2, Y_{0}\right)$ appartient à la cubique et on l'appelle l'origine de la cubique. Les cubiques limites associées aux paires exceptionnelles sont spéciales et les origines de ces cubiques sont les points limites correspondants. Ces cubiques ont toutes le même terme de plus haut degré, c'est $-\frac{1}{6} \Delta_{1}^{3}$. On considère l'automorphisme de $\mathbb{R}^{2}$

$$
\lambda:(X, Y) \longmapsto\left(X, Y+\frac{1}{6} X^{3}\right) .
$$


L’image de la cubique précédente par $\lambda$ est la conique d'équation

$$
Y=\frac{X_{0}}{2} \cdot X^{2}+\left(\beta-\frac{X_{0}^{2}}{2}\right) \cdot X+\frac{X_{0}^{3}}{6}-\beta X_{0}+Y_{0} .
$$

Si la cubique est spéciale, la conique est aussi dite spéciale et son origine est le point $\left(X_{0}-2, Y_{0}+\frac{1}{6}\left(X_{0}-2\right)^{3}\right)$.

On suppose que $\mathbb{R}^{2} \subset \mathbb{P}_{2}$, ce dernier étant muni des coordonnées $X, Y, Z$, le point de $\mathbb{P}_{2}$ correspondant à $(X, Y) \in \mathbb{R}^{2}$ étant $(X, Y, 1)$. Soit $W$ le sous-espace vectoriel de $H^{0}\left(\mathbb{P}_{2}, \mathcal{O}(2)\right)$ constitué des formes quadratiques du type

$$
u \cdot Y Z+v \cdot X^{2}+w \cdot X Z+t \cdot Z^{2} .
$$

C'est donc l'espace des équations de coniques passant par $(0,1,0)$ et tangentes à la droite d'équation $Z=0$ en ce point. Les coniques de $\mathbb{R}^{2}$ considérées précédemment sont des traces sur $\mathbb{R}^{2}$ de coniques de $W$. Les coniques spéciales sont les points de la quadrique d'équation

$$
w u+\frac{2}{3} u^{2}-2 v^{2}=0 .
$$

On munit $\mathbb{P}(W)$ des coordonnées $Y Z, X^{2}, X Z, Z^{2}$. Le morphisme rationnel associant à un point du plan la conique spéciale passant par ce point est

$$
\begin{aligned}
\sigma: \mathbb{P}_{2} & \longrightarrow \mathbb{P}(W) \\
(x, y, z) & \longmapsto\left(-z^{2}, \frac{x z}{2}+z^{2},-\frac{x^{2}}{2}-2 x z-\frac{4}{3} z^{2}, y z+x^{2}+\frac{4}{3} x z\right) .
\end{aligned}
$$

On voit aisément que $\sigma$ vérifie les conditions $1,2,3 \mathrm{du} \S 2.3$. La matrice de l'application $\phi=\left(\sigma^{*}\right)^{-1}: W \longrightarrow W^{*}$ relativement à la base $\left(Y Z, X^{2}, X Z, Z^{2}\right)$ est

$$
\left(\begin{array}{cccc}
\frac{8}{3} & -\frac{16}{3} & 2 & -1 \\
\frac{16}{3} & -8 & 2 & 0 \\
2 & -2 & 0 & 0 \\
1 & 0 & 0 & 0
\end{array}\right)
$$

Soit $\alpha=\left(E_{0}, E_{1}, E_{2}, E_{3}\right)$ un base d'hélice de fibrés exceptionnels sur $\mathbb{P}_{3}$. On note $u(\alpha)$ (resp. $v(\alpha))$ le plan de $W$ engendré par les coniques $\lambda\left(C\left(E_{0}\right)\right)$ et $\lambda\left(C\left(E_{1}\right)\right)$ (resp. $\lambda\left(C\left(E_{1}\right)\right)$ et $\lambda\left(C\left(E_{2}\right)\right)$ ) (ou plus éxactement par les coniques de $\mathbb{P}_{2}$ engendrées par ces coniques réelles).

Proposition 3.3. 1) L'application

$$
\begin{array}{ccc}
\Lambda: B\left(\mathbb{P}_{3}\right) & \longrightarrow & G_{2,4} \times G_{2,4} \\
\alpha & \longmapsto & (u(\alpha), v(\alpha))
\end{array}
$$

est à valeurs dans $Z(\phi)$.

2) On a $\tau(\phi) \circ \Lambda=\Lambda \circ \tau$ et $L_{1} \circ \Lambda=\Lambda \circ L$.

Démonstration. Soient $\left(F_{i}\right)_{i \in \mathbb{Z}}$ la suite de fibrés exceptionnels associée à la paire $\left(E_{2}, E_{3}\right)$, $P_{-}=\lim _{i \rightarrow-\infty} P\left(F_{i}\right), P_{+}=\lim _{i \rightarrow+\infty} P\left(F_{i}\right)$. On a pour tout entier $i, \chi\left(F_{i}, E_{0}\right)=\chi\left(F_{i}, E_{1}\right)=0$, donc $P\left(F_{i}\right) \in S\left(E_{0}\right) \cap S\left(E_{1}\right)$, et par passage à la limite, on a $P_{-}, P_{+} \in C\left(E_{0}\right) \cap C\left(E_{1}\right)$. Comme ces points sont distincts, on a

$$
C\left(E_{0}\right) \cap C\left(E_{1}\right)=\left\{P_{-}, P_{+},(0,1,0)\right\},
$$


et $u(\alpha)$ est éxactement la droite projective des coniques de $\mathbb{P}(W)$ passant par $\lambda\left(P_{-}\right)$et $\lambda\left(P_{+}\right)$. Soient $\left(G_{i}\right)_{i \in \mathbb{Z}}$ la suite de fibrés exceptionnels associée à la paire $\left(E_{0}, E_{1}\right), Q_{-}=\lim _{i \rightarrow-\infty} P\left(G_{i}\right)$, $Q_{+}=\lim _{i \rightarrow+\infty} P\left(G_{i}\right)$. Alors ce qui précède montre que pour tout entier $i$ on a aussi

$$
C\left(G_{i}\right) \cap C\left(G_{i+1}\right)=\left\{Q_{-}, Q_{+},(0,1,0)\right\}
$$

d'où on déduit que

$$
\lambda\left(C\left(E_{0}\right)\right) \wedge \lambda\left(C\left(E_{1}\right)\right)=\lambda\left(C\left(Q_{-}\right)\right) \wedge \lambda\left(C\left(Q_{+}\right)\right) .
$$

De même, on a $\lambda\left(C\left(E_{2}\right)\right) \wedge \lambda\left(C\left(E_{3}\right)\right)=\lambda\left(C\left(P_{-}\right)\right) \wedge \lambda\left(C\left(P_{+}\right)\right)$. Il découle donc de la proposition 2.7 que

$$
\xi(\phi)(u(\alpha))=\lambda\left(C\left(E_{2}\right)\right) \wedge \lambda\left(C\left(E_{3}\right)\right)
$$

On a donc

$$
u(\alpha) \cap v(\alpha)=\lambda\left(C\left(E_{1}\right)\right), v(\alpha) \cap \xi(\phi)(u(\alpha))=\lambda\left(C\left(E_{2}\right)\right),
$$

ce qui démontre 1).

La démonstration de 2) est analogue.

Pour finir on montre qu'on obtient finalement la même construction qu'au § 2.5.

Proposition 3.4. Il existe un isomorphisme $\eta: W_{K} \longrightarrow W$ tel que $\phi_{K}={ }^{t} \eta \circ \phi \circ \eta$ et si $\eta^{\prime}: Z\left(\phi_{K}\right) \longrightarrow Z(\phi)$ est l'isomorphisme déduit de $\eta$ on a un diagramme commutatif

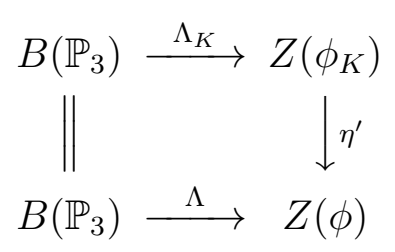

Démonstration. On suppose que $W_{K}$ est muni de la base $\left(f_{0}, f_{1}, f_{2}, f_{3}\right)$ du $\S 2.5$, et $W$ de la base $\left(Y Z, X^{2}, X Z, Z^{2}\right)$. L'application $\eta$ a pour matrice

$$
\left(\begin{array}{cccc}
1 & 3 & 3 & 1 \\
1 & \frac{5}{2} & 2 & \frac{1}{2} \\
\frac{19}{12} & \frac{9}{4} & \frac{3}{4} & \frac{1}{12} \\
\frac{1}{2} & -\frac{5}{4} & -1 & -\frac{1}{4}
\end{array}\right)
$$

relativement à ces bases. 


\section{EQUATIONS VÉRIFIÉES PAR LES INVARIANTS DES FIBRÉS EXCEPTIONNELS}

\subsection{Sous-variétés de $G_{2,4}$}

On se place sous les hypothèses du $\S 2.1$, et on suppose que $\xi(\phi) \circ \xi(\phi) \neq-I_{G_{2,4}}$. Soient $u, v \in G_{2,4}$, vus comme des droites de $\mathbb{P}(W)$. Si $u \cap v \neq \emptyset$, on note $Z(u, v)$ l'ensemble des droites de $\mathbb{P}(W)$ rencontrant $u$ et $v$. C'est une surface de $G_{2,4}$.

Lemme 4.1. L'ensemble $\{u, v\}$ est entièrement déterminé par $Z(u, v)$.

Démonstration. En effet, $u \cup v$ est le lieu des points de $\mathbb{P}(W)$ qui sont des intersections d'éléments de $Z(u, v)$.

On notera plus simplement $Z_{u}=Z(u, \xi(\phi)(u))$ si $u . \xi(\phi(u)) \neq 0$.

Soient $P \subset \mathbb{P}(W)$ un plan projectif et $x \in P$. Soit $L$ l'ensemble des droites de $\mathbb{P}(W)$ contenant $x$ et contenues dans $\mathbf{P}$. C'est une droite de $G_{2,4}$. Soit $V_{L}$ l'adhérence de l'union des $Z_{u}, u$ parcourant $L$. C'est un volume de $G_{2,4}$, d'après le lemme précédent.

Lemme 4.2. Le plan $\mathbf{P}$ et le point $x$ sont entièrement déterminés par $V_{L}$.

Démonstration. Soient $A$ et $B$ des éléments de $\wedge^{2} W$ tels que $a=\mathbb{C} . A$ et $b=\mathbb{C} . B$ engendrent $\mathbf{P}$ et passent par $x$. Soit

$$
\bar{\xi}: \wedge^{2} W \longrightarrow \wedge^{2} W
$$

un automorphisme au dessus de $\xi(\phi)$. On peut supposer que $\bar{\xi}$ est orthogonal respectivement à la forme quadratique $j$ sur $\wedge^{2} W$. Alors, si $d \in G_{2,4}$, on a $d \in V_{L}$ si et seulement si il existe des scalaires non tous nuls $\lambda$ et $\mu$ tels que

$$
D \cdot(\lambda \cdot A+\mu \cdot B)=D \cdot(\lambda \cdot \bar{\xi}(A)+\mu \cdot \bar{\xi}(B))=0,
$$

ce qui équivaut à

$$
\left|\begin{array}{cc}
D . A & D \cdot B \\
D \cdot \bar{\xi}(A) & D \cdot \bar{\xi}(B)
\end{array}\right|=0 .
$$

Soit $\left(e_{0}, \ldots, e_{5}\right)$ une base orthogonale de $\wedge^{2} W$ et posons

$$
\begin{gathered}
A=\sum_{0 \leq i \leq 5} a_{i} \cdot e_{i}, B=\sum_{0 \leq i \leq 5} b_{i} \cdot e_{i}, D=\sum_{0 \leq i \leq 5} d_{i} \cdot e_{i}, \\
f_{i}=\bar{\xi}\left(e_{i}\right), 0 \leq i \leq 5 .
\end{gathered}
$$

Alors on a $d \in V_{L}$ si et seulement si

$$
\sum_{0 \leq i, j \leq 5} \lambda_{i j} \cdot d_{i} d_{j}=0
$$

avec

$$
\lambda_{i j}=\sum_{0 \leq k \leq 5}\left(a_{i} b_{k}-a_{k} b_{i}\right) e_{k} \cdot f_{j} .
$$

Autrement dit, $V_{L}$ est l'intersection de $G_{2,4}$ avec la quadrique de $\mathbb{P}\left(\wedge^{2} W\right)$ définie par l'équation

$$
\sum_{0 \leq i \leq 5} \lambda_{i j} \cdot X_{i} X_{j}=0 .
$$


Toute quadrique de $\mathbb{P}\left(\wedge^{2} W\right)$ contenant $V_{L}$ a une équation qui est une combinaison linéaire de la précédente et de

$$
\sum_{0 \leq i \leq 5} X_{i}^{2}=0
$$

Supposons que $V_{L}=V_{L^{\prime}}, L^{\prime}$ étant engendrée par $\mathbb{C} . A^{\prime}$ et $\mathbb{C} . B^{\prime}$, avec

$$
A^{\prime}=\sum_{0 \leq i \leq 5} a_{i}^{\prime} \cdot e_{i}, B^{\prime}=\sum_{0 \leq i \leq 5} b^{\prime} i_{i} e_{i} .
$$

Il existe alors des scalaires $s, t$ tels que

$$
\left(a_{i}^{\prime} b_{j}^{\prime}-a_{j}^{\prime} b_{i}^{\prime}\right) \cdot\left(e_{i} \cdot f_{j}\right)=s \cdot I_{5}+t \cdot\left(a_{i} b_{j}-a_{j} b_{i}\right) \cdot\left(e_{i} \cdot f_{j}\right) .
$$

D'où on déduit que $s .\left(e_{i} \cdot f_{j}\right)^{-1}$ est une matrice antisymétrique. Si $s \neq 0$, ceci implique que $\bar{\xi} \circ \bar{\xi}=-I_{G_{2,4}}$, contrairement à l'hypothèse. On a donc $s=0$, d'où

$$
\left(a_{i}^{\prime} b_{j}^{\prime}-a_{j}^{\prime} b_{i}^{\prime}\right)=t .\left(a_{i} b_{j}-a_{j} b_{i}\right)
$$

et $L=L^{\prime}$.

\subsection{La sous-variété de $Z\left(\phi_{K}\right)$ engendrée par $B\left(\mathbb{P}_{3}\right)$}

Soit Exc le sous-ensemble de $\mathbb{P}\left(W_{K}\right)$ constitué des points associés aux fibrés exceptionnels .

A toute paire exceptionnelle $\left(E_{0}, E_{1}\right)$ on associe l'élément $\mathbb{C}$. $\left[E_{0}\right] \oplus \mathbb{C}$. [ $\left.E_{1}\right]$ de $G_{2,4}$. Il ne dépend en fait que de la suite $\left(F_{i}\right)_{i \in \mathbb{Z}}$ de fibrés exceptionnels associée à $\left(E_{0}, E_{1}\right)$. On note note $S E x c$ le sous-ensemble de $G_{2,4}$ constitué de ces points.

Soit $H\left(\phi_{K}\right)$ la sous-variété fermé de $\mathbb{P}\left(W_{K}\right) \times \mathbb{P}\left(W_{K}\right)$ constituée des $(x, y)$ tels que $\chi(x, y)=x \cdot \phi_{K}(y)=0$. A toute paire exceptionnelle $\left(E_{0}, E_{1}\right)$ on associe le point $\left(\left[E_{1}\right],\left[E_{0}\right]\right)$ de $H\left(\phi_{K}\right)$. On note PExc le sous-ensemble de $H\left(\phi_{K}\right)$ constitué de ces points.

On rappelle enfin qu'on peut voir l'ensemble $B\left(\mathbb{P}_{3}\right)$ des bases d'hélices de fibrés exceptionnels sur $\mathbb{P}_{3}$ comme un sous-ensemble de $Z\left(\phi_{K}\right)$.

Proposition 4.3. On a, pour la topologie de Zariski :

(i) Exc est dense dans $\mathbb{P}\left(W_{K}\right)$,

(ii) Sexc est dense dans $G_{2,4}$,

(iii) $B\left(\mathbb{P}_{3}\right)$ est dense dans $Z\left(\phi_{K}\right)$,

Pexc est dense dans $H\left(\phi_{K}\right)$.

Démonstration. Démontrons d'abord (ii). Soit $X$ l'adhérence de $S E x c$ dans $G_{2,4}$. Soit $\left(E_{0}, E_{1}, E_{2}, E_{3}\right)$ une base d'hélice. Posons $u=\mathbb{C}$. $\left[E_{0}\right] \oplus \mathbb{C}$. [ $\left.E_{1}\right]$. Alors on a

$\xi\left(\phi_{K}\right)(u)=\mathbb{C}$. $\left[E_{2}\right] \oplus \mathbb{C}$. $\left[E_{3}\right]$. Soit $\left(F_{i}\right)_{i \in \mathbb{Z}}\left(\right.$ resp. $\left.\left(G_{i}\right)_{i \in \mathbb{Z}}\right)$ la suite de fibrés exceptionnels associée à $\left(E_{0}, E_{1}\right)$ (resp. $\left.\left(E_{2}, E_{3}\right)\right)$, c'est-à-dire que $F_{0}=E_{0}, F_{1}=E_{1}$ (resp. $\left.G_{0}=E_{2}, G_{1}=E_{3}\right)$ et que pour tout entier $i$ on a une suite éxacte

$$
\begin{gathered}
0 \longrightarrow F_{i-1} \longrightarrow F_{i} \otimes \operatorname{Hom}\left(F_{i}, F_{i+1}\right) \longrightarrow F_{i+1} \longrightarrow 0 \\
\left(\text { resp. } 0 \longrightarrow G_{i-1} \longrightarrow G_{i} \otimes \operatorname{Hom}\left(G_{i}, G_{i+1}\right) \longrightarrow G_{i+1} \longrightarrow 0\right)
\end{gathered}
$$


Alors, pour tous entiers $k, l$,

$$
u_{k l}=\mathbb{C} \cdot\left[F_{k}\right] \oplus \mathbb{C} \cdot\left[G_{l}\right]
$$

est un point de $Z_{u}$, car on a

$$
u \cap u_{k l}=\mathbb{C} \cdot\left[F_{k}\right] \text { et } \xi\left(\phi_{K}\right)(u) \cap u_{k l}=\mathbb{C} \cdot\left[G_{l}\right] .
$$

Il en découle que pour tout entier $k, X$ contient la courbe constituée des droites passant par $\mathbb{C}$. $\left[F_{k}\right]$ et rencontrant $\xi\left(\phi_{K}\right)(u)$. D'où on déduit que $Z_{u} \subset X$. Soit $k$ un entier. Puisque $Z_{u_{k l}} \subset X$ pour tout $l$, on a d'après le lemme $4.1, V_{L_{k}} \subset X, L_{k}$ désignant la droite de $G_{2,4}$ constituée des droites de $\mathbb{P}(W)$ passant par $\mathbb{C}$. $\left[F_{k}\right]$ et rencontrant $\xi\left(\phi_{K}\right)(u)$. D'après le lemme 4.2 , il en découle que $X$ est de dimension au moins 4. Donc $X=G_{2,4}$, ce qui démontre (ii).

Démontrons maintenant (i). Soit $Y$ l'adhérence de Exc dans $\mathbb{P}\left(W_{K}\right)$. Alors, pour tout entier $k, Y$ contient le point $\mathbb{C}$. $\left[F_{k}\right]$ de la droite $u$. Donc $u \subset Y$. Soit $k$ un entier. Alors, pour tout entier $l, Y$ contient la droite $\mathbb{C} .\left[F_{k}\right] \oplus \mathbb{C}$. $\left[G_{l}\right]$, donc $Y$ contient le plan $P_{k}$ engendré par $\mathbb{C}$. $\left[F_{k}\right]$ et $\xi\left(\phi_{K}\right)(u)$. Puisque $Y$ contient tous les plans $P_{k}$, on a $Y=\mathbb{P}\left(W_{K}\right)$, ce qui démontre (i).

Prouvons maintenant (iii). Soient $T$ l'adhérence de $B\left(\mathbb{P}_{3}\right)$ dans $Z\left(\phi_{K}\right)$ et $\pi$ la restriction à $T$ de la première projection

$$
Z\left(\phi_{K}\right) \longrightarrow G_{2,4}
$$

D'après la démonstration de (ii), la fibre de $\pi$ au dessus de $u$ est $Z_{u}$, c'est-à-dire que cette fibre est de dimension maximale. Puisque la sous-variété fermée de $T$ constituée des points $z$ tels que $\pi^{-1}(\pi(z))$ soit de dimension maximale contient $B\left(\mathbb{P}_{3}\right)$, cette sous-variété contient $T$, c'est-à-dire que $T=\pi^{-1}(X)$. Comme $X=G_{2,4}$ d'après (ii), on a $T=Z\left(\phi_{K}\right)$, ce qui démontre (iii).

Il reste à démontrer (iv). Soit $R$ l'adhérence de $P E x c$ dans $H\left(\phi_{K}\right)$. On note $\alpha$ la restriction à $R$ du morphisme rationnel

$$
H\left(\phi_{K}\right) \longrightarrow G_{2,4}
$$

associant à $(\mathrm{x}, \mathrm{y})$ le plan $x \oplus y($ si $x \neq y)$. D'après (ii), $\alpha$ est surjectif. Au dessus de $u$, la fibre de $\alpha$ contient les points $\mathbb{C}$. $\left[F_{i}\right] \oplus \mathbb{C}$. $\left[F_{i+1}\right]$, et est donc infinie. Donc elle est de dimension au moins 1. En raisonnant comme dans la démonstration de (iii), on voit que les fibres de $\alpha$ sont de dimension au moins 1 . Donc $R$ est de dimension au moins 5 . Comme $\operatorname{dim}\left(H\left(\phi_{K}\right)\right)=5$, on a $R=H\left(\phi_{K}\right)$, ce qui démontre (iv).

\subsection{Equations algébriques vérifiées par les invariants des fibrés exceptionnels}

Fixons d'abord certaines notations.

Les applications

$$
r g, c_{1}, c_{2}, c_{3}: K\left(\mathbb{P}_{3}\right) \longrightarrow \mathbb{Z}
$$

se prolongent en des fonctions régulières sur $W_{K}$, qu'on note de la même façon. Le morphisme

$$
\begin{aligned}
\theta: W_{K} & \longrightarrow \mathbb{C}^{4} \\
x & \longmapsto\left(r g(x), c_{1}(x), c_{2}(x), c_{3}(x)\right)
\end{aligned}
$$

est un isomorphisme (non linéaire). 
Le morphisme

$$
\begin{aligned}
\mathbb{C}^{4} & \longrightarrow \mathbb{C}^{4} \\
\left(r, c_{1}, c_{2}, c_{3}\right) & \longmapsto\left(r, \Delta_{1}, \Delta_{2}, \Delta_{3}\right),
\end{aligned}
$$

où $\Delta_{1}, \Delta_{2}, \Delta_{3}$ sont donnés par les formules du $\S 1.1$, est un isomorphisme birationnel. Son inverse est le morphisme

$$
\left(r, \Delta_{1}, \Delta_{2}, \Delta_{3}\right) \longmapsto\left(r, C_{1}, C_{2}, C_{3}\right)
$$

avec

$$
\begin{gathered}
C_{1}=r . \Delta_{1}, C_{2}=\frac{r(r-1)}{2} \cdot \Delta_{1}^{2}-r . \Delta_{2}, \\
C_{3}=\frac{r(r-1)(r-2)}{6} \cdot \Delta_{1}^{3}+r(r-2) \cdot \Delta_{1} \Delta_{2}+2 r . \Delta_{3} .
\end{gathered}
$$

On pose $\delta_{i}=\Delta_{i} \circ \theta, 1 \leq i \leq 3$. On a donc, pour tout fibré vectoriel $E$ sur $\mathbb{P}_{3}, \delta_{i}([E])=\Delta_{i}(E)$. Ce sont des fonctions rationnelles sur $W_{K}$, définies en dehors de l'hyperplan d'équation $r=0$. On a, pour tous $t \in \mathbb{C}^{*}, x \in W_{K}$ tel que $r g(x) \neq 0$,

$$
\delta_{i}(t x)=\delta_{i}(x) .
$$

Les fonctions $\delta_{i}$ définissent donc des fonctions rationnelles $\delta_{i}^{\prime}$ sur $\mathbb{P}\left(W_{K}\right)$, et

$$
\begin{aligned}
\mu: \mathbb{P}\left(W_{K}\right) & \longrightarrow \mathbb{C}^{3} \\
x & \longmapsto\left(\delta_{1}^{\prime}(x), \delta_{2}^{\prime}(x), \delta_{3}^{\prime}(x)\right)
\end{aligned}
$$

est un isomorphisme birationnel dont l'inverse est régulier.

\section{Démonstration du théorème $C$ :}

On utilise les notations de l'Introduction.

Soit $P \in R^{\prime \prime}$ tel que pour tout fibré exceptionnel $E$ sur $\mathbb{P}_{3}$ on ait

$$
P\left(r g(E), \Delta_{1}(E), \Delta_{2}(E), \Delta_{3}(E)\right)=0 .
$$

On peut écrire $P$ sous la forme

$$
P\left(R_{0}, \Delta_{10}, \Delta_{20}, \Delta_{30}\right)=R_{0} \cdot P_{1}\left(R_{0}^{2}, \Delta_{10}, \Delta_{20}, \Delta_{30}\right)+P_{2}\left(R_{0}^{2}, \Delta_{10}, \Delta_{20}, \Delta_{30}\right),
$$

$P_{1}$ et $P_{2}$ étant des polynômes. Soit

$$
Q\left(X, \Delta_{10}, \Delta_{20}, \Delta_{30}\right)=X . P_{1}\left(X, \Delta_{10}, \Delta_{20}, \Delta_{30}\right)^{2}-P_{2}\left(X, \Delta_{10}, \Delta_{20}, \Delta_{30}\right)^{2} .
$$

Alors on a, pour tout fibré exceptionnel $E$ sur $\mathbb{P}_{3}$

$$
Q\left(r g(E)^{2}, \Delta_{1}(E), \Delta_{2}(E), \Delta_{3}(E)\right)=0 .
$$

Soit $Y$ la sous-variété de $\mathbb{C}^{4}$ définie par $\mathcal{I}^{\prime \prime}$. C'est l'hypersurface intègre d'équation $R_{0}^{2} \cdot\left(1-4 \Delta_{20}\right)=1$. On pose

$$
Q\left(\frac{1}{1-4 \Delta_{20}}, \Delta_{10}, \Delta_{20}, \Delta_{30}\right)=\frac{S\left(\Delta_{10}, \Delta_{20}, \Delta_{30}\right)}{\left(1-4 \Delta_{20}\right)^{k}},
$$

$S$ étant un polynôme et $k$ un entier convenable. Alors, pour tout fibré exceptionnel $E$ sur $\mathbb{P}_{3}$, on a

$$
S\left(\Delta_{1}(E), \Delta_{2}(E), \Delta_{3}(E)\right)=0
$$


On a donc $S \circ \mu=0$ sur Exc, et d'après la proposition 4.3, ceci implique que $S \circ \mu=0$, d'où $S=0$. L'intégrité de $Y$ entraîne que $Q\left(R_{0}^{2}, \Delta_{10}, \Delta_{20}, \Delta_{30}\right) \in \mathcal{I}^{\prime \prime}$. Donc, $\mathcal{I}^{\prime \prime}$ étant premier, on a $P \in \mathcal{I}^{\prime \prime}$ ou $R_{0} \cdot P_{1}\left(R_{0}^{2}, \Delta_{10}, \Delta_{20}, \Delta_{30}\right)-P_{2}\left(R_{0}^{2}, \Delta_{10}, \Delta_{20}, \Delta_{30}\right) \in \mathcal{I}^{\prime \prime}$. Dans le second cas, on voit que pour tout fibré exceptionnel $E$ sur $\mathbb{P}_{3}$ on a

$$
P_{i}\left(r g(E)^{2}, \Delta_{1}(E), \Delta_{2}(E), \Delta_{3}(E)\right)=0, i=1,2,
$$

d'où, par le même raisonnement que précédemment on déduit que $P_{1}, P_{2} \in \mathcal{I}^{\prime \prime}$, et finalement aussi $P \in \mathcal{I}^{\prime \prime}$. Ceci prouve le théorème $\mathrm{C}$.

Démonstration des théorèmes $A$ et $B$ :

On ne démontrera que le théorème $\mathrm{A}$, le théorème $\mathrm{B}$ étant analogue.

Soit $Y$ la sous-variété fermée de $\mathbb{C}^{16}$ qui est la variété réduite associée à la sous-variété définie par l'idéal $\mathcal{I}$. On va définir un morphisme rationnel

$$
\rho: Z\left(\phi_{K}\right) \longrightarrow Y \text {. }
$$

Soit $(u, v)$ un point général de $Z\left(\phi_{K}\right)$. Posons

$$
l_{0}=\xi\left(\phi_{K}\right)^{-1}(v) \cap u, l_{1}=u \cap v, l_{2}=v \cap \xi\left(\phi_{K}\right)(u), l_{3}=\xi\left(\phi_{K}\right)(u) \cap \xi\left(\phi_{K}\right)(v),
$$

qui sont des points de $\mathbb{P}\left(W_{K}\right)$. On pose

$$
\rho(u, v)=\left(\left(\frac{1}{1-4 \delta_{2}^{\prime}\left(l_{i}\right)}\right)_{0 \leq i \leq 3},\left(\left(\delta_{j}^{\prime}\left(l_{i}\right)_{1 \leq j \leq 3}\right)_{0 \leq i \leq 3}\right),\right.
$$

qui est un point de $Y$ (l'équation $\chi_{10}=0$ par exemple équivaut à $l_{1} \subset l_{0}^{\perp}$, qui est vrai car $l_{0} \in \xi\left(\phi_{K}\right)^{-1}(v)$ et $\left.l_{1} \in v=\xi\left(\phi_{K}\right)^{-1}(v)^{\perp}\right)$.

Lemme 4.4. Le morphisme $\rho$ est un isomorphisme birationnel.

Démonstration. Considérons le morphisme rationnel $\gamma: Y \longrightarrow Z\left(\phi_{K}\right)$ qui au point $\left(\left(X_{i}\right)_{0 \leq i \leq 3},\left(\left(\Delta_{i j}\right)_{1 \leq i \leq 3}\right)_{0 \leq j \leq 3}\right)$ de $Y$ associe l'élément

$$
\left(\mu^{-1}\left(\Delta_{10}, \Delta_{20}, \Delta_{30}\right) \oplus \mu^{-1}\left(\Delta_{11}, \Delta_{21}, \Delta_{31}\right), \mu^{-1}\left(\Delta_{11}, \Delta_{21}, \Delta_{31}\right) \oplus \mu^{-1}\left(\Delta_{12}, \Delta_{22}, \Delta_{32}\right)\right)
$$

de $Z\left(\phi_{K}\right)$. Il suffit de prouver que le lieu $T$ des points de $Y$ où $\mu$ n'est pas défini est de dimension au plus 5 .

On a

avec

$$
T=\left(\bigcup_{0 \leq k \leq 3} T_{k}\right) \cup\left(\bigcup_{0 \leq k<l \leq 3} T_{k l}\right),
$$

$$
\begin{aligned}
T_{k} & =\left\{\left(\left(X_{i}\right),\left(\left(\Delta_{i j}\right)\right)\right) \in Y, \Delta_{1 k}=\Delta_{2 k}=\Delta_{3 k}=0\right\}, \\
T_{k l} & =\left\{\left(\left(X_{i}\right),\left(\left(\Delta_{i j}\right)\right)\right) \in Y,\left(\Delta_{i k}\right)_{1 \leq i \leq 3}=\left(\Delta_{i l}\right)_{1 \leq i \leq 3}\right\} .
\end{aligned}
$$

On a $T_{k l}=\emptyset$, car les équations $\chi_{k l}=0$ et $\chi_{k k}=0$ sont incompatibles sur la variété des points $\left(\left(X_{i}\right),\left(\left(\Delta_{i j}\right)\right)\right)$ tels que $\Delta_{i k}=\Delta_{i l}$ pour $1 \leq i \leq 3$.

Montrons que $\operatorname{dim}\left(T_{0}\right)<6$ (les autres cas sont analogues). Considérons la sous-variété $X$ de $\mathbb{C}^{16}$ constituée des points $\left(\left(X_{i}\right),\left(\left(\Delta_{i j}\right)\right)\right)$ tels que $\chi_{i i}\left(X_{i}, \Delta_{2 i}\right)=0$ pour $0 \leq i \leq 3$ et 
$\Delta_{10}=\Delta_{20}=\Delta_{30}=0$, qui est irréductible et de dimension 9 . Les équations $\chi_{10}=\chi_{20}=$ $\chi_{30}=0$ définissent une sous-variété $X^{\prime}$ de dimension 6 de $X$ car elles font intervenir des variables différentes. Comme elles reviennent à exprimer les variables $\Delta_{3 i}, 1 \leq i \leq 3$, en fonction des autres variables, $X^{\prime}$ est irréductible. Les autres équations introduisent des relations supplémentaires entre les autres variables. On a donc $\operatorname{dim}\left(T_{0}\right)<6$.

Il découle du lemme précédent que $Y$ est irréductible. Le reste de la démonstration du théorème $\mathrm{A}$ est maintenant analogue à celle du théorème $\mathrm{C}$.

\section{RÉFÉRENCES}

[1] Drézet, J.-M.Fibrés exceptionnels et suite spectrale de Beilinson sur $\mathbb{P}_{2}(\mathbb{C})$. Math. Ann. 275 (1986), 25-48.

[2] Drézet, J.-M.Structures géométriques associées aux hélices de fibrés exceptionnels. Preprint Univ. Paris 7 (1992).

[3] Drézet, J.-M., Le Potier, J.Fibrés stables et fibrés exceptionnels sur $\mathbb{P}_{2}$. Ann. Ec. Norm. Sup. 18 (1985), 193-244.

[4] Gorodentsev, A.L.Exceptional bundles on surfaces with a moving anticanonical class. Math. USSR Izvestiya. AMS transl. 33 (1989), 67-83.

[5] Gorodentsev, A.L, Rudakov, A.N.Exceptional vector bundles on projective spaces. Duke Math. Journ. 54 (1987), 115-130.

[6] Nogin, D.Y.Helices of period 4 and Markov type equations. A paraître dans Math. USSR Izvestiya. AMS transl.

[7] Nogin, D.Y.Helices on some Fano threefolds : constructivity of semi-orthogonal bases of $K_{0}$. Ann. Scient. Ec. Norm. Sup. 27 (1994),1-44.

[8] Rudakov, A.N.Exceptional vector bundles on a quadric. Math. USSR Izvestiya. AMS transl. 33 (1989), 115-138.

[9] Rudakov, A.N.A description of Chern classes of semi-stable sheaves on a quadric surface. J. reine angew. Math. 453 (1994), 113-135.

Institut de Mathématiques de Jussieu, Case 247, 4 Place Jussieu, F-75252 Paris, France

E-mail address: drezet@math.jussieu.fr

$U R L:$ http://www.math.jussieu.fr/ $\sim$ drezet 